\title{
Supplementary
}

\section{Cytotoxic illudane sesquiterpenes from the fungus Granulobasidium vellereum (Ellis \& Cragin) Jülich}

Christina Nord, ${ }^{\dagger}$ Audrius Menkis, ${ }^{\ddagger}$ Anders Broberg ${ }^{*}$,

†Department of Chemistry and Biotechnology, Uppsala BioCenter, Swedish University of Agricultural Sciences, P.O. Box 7015, SE-75007, Uppsala, Sweden.

${ }^{\ddagger}$ Department of Forest Mycology and Plant Pathology, Uppsala BioCenter, Swedish University of Agricultural Sciences, P.O. Box 7026, SE-75007, Uppsala, Sweden. 


\section{Contents}

Figure S1: ${ }^{1}$ H NMR (methanol-d4, $600 \mathrm{MHz}$ ) spectrum of 1...................................................... S4

Figure S2: ${ }^{13} \mathrm{C}$ NMR (methanol-d4, $150 \mathrm{MHz}$ ) spectrum of 1. ..................................................... S4

Figure S3: ${ }^{1} \mathrm{H}$ NMR (chloroform-d, $600 \mathrm{MHz}$ ) spectrum of 1.................................................... 55

Figure S4: ${ }^{1} \mathrm{H}$ NMR (methanol-d4, $600 \mathrm{MHz}$ ) spectrum of 2...................................................... S5

Figure S5: ${ }^{13} \mathrm{C}$ NMR (methanol-d4, $150 \mathrm{MHz}$ ) spectrum of 2. .................................................... S6

Figure S6: ${ }^{1} \mathrm{H}$ NMR (chloroform- $d, 600 \mathrm{MHz}$ ) spectrum of 2. ....................................................... 66

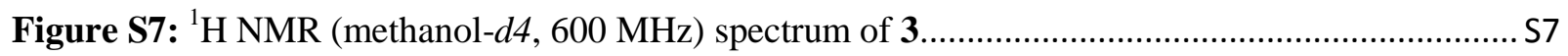

Figure S8: ${ }^{13} \mathrm{C}$ NMR (methanol-d4, $150 \mathrm{MHz}$ ) spectrum of 3. ....................................................... S7

Figure S9: ${ }^{1} \mathrm{H}$ NMR (chloroform-d, $600 \mathrm{MHz}$ ) spectrum of 3. ...................................................... S8

Figure S10: ${ }^{1} \mathrm{H}$ NMR (methanol-d4, $600 \mathrm{MHz}$ ) spectrum of 4...................................................... S8

Figure S11: ${ }^{13} \mathrm{C}$ NMR (methanol-d4, $150 \mathrm{MHz}$ ) spectrum of 4.................................................. S9

Figure S12: ${ }^{1} \mathrm{H}$ NMR (chloroform- $d, 600 \mathrm{MHz}$ ) spectrum of 4 . ................................................... S9

Figure S13: ${ }^{1}$ H NMR (methanol-d4, $600 \mathrm{MHz}$ ) spectrum of 5................................................... S10

Figure S14: ${ }^{13} \mathrm{C}$ NMR (methanol-d4, $150 \mathrm{MHz}$ ) spectrum of 5. ................................................ S10

Figure S15: ${ }^{1} \mathrm{H}$ NMR (acetone-d6, $600 \mathrm{MHz}$ ) spectrum of 5..................................................... S11

Figure S16: ${ }^{1} \mathrm{H}$ NMR (DMSO-d6, $600 \mathrm{MHz}$ ) spectrum of 5....................................................... S11

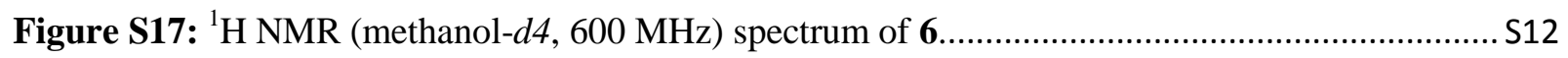

Figure S18: ${ }^{13} \mathrm{C}$ NMR (methanol-d4, $150 \mathrm{MHz}$ ) spectrum of 6. ................................................... S12

Figure S19: ${ }^{1} \mathrm{H}$ NMR (acetone-d6, $600 \mathrm{MHz}$ ) spectrum of 6.................................................. S13

Figure S20: ${ }^{1} \mathrm{H}$ NMR (pyridine-d5, $600 \mathrm{MHz}$ ) spectrum of the (S)-MTPA ester of 1..................... S13

Figure S21: ${ }^{1} \mathrm{H}$ NMR (pyridine-d5, $600 \mathrm{MHz}$ ) spectrum of the (R)-MTPA ester of 1..................... S14

Figure S22: ${ }^{1}$ H NMR (methanol-d4, $600 \mathrm{MHz}$ ) spectrum of 2a................................................... S14

Figure S23: ${ }^{1} \mathrm{H}$ NMR (pyridine-d5, $600 \mathrm{MHz}$ ) spectrum of the (S)-MTPA ester of 2..................... S15

Figure S24: ${ }^{1} \mathrm{H}$ NMR (pyridine-d5, $600 \mathrm{MHz}$ ) spectrum of the (R)-MTPA ester of 2..................... S15

Figure S25: ${ }^{1} \mathrm{H}$ NMR (methanol-d4, $600 \mathrm{MHz}$ ) spectrum of 3a................................................. S16

Figure S26: ${ }^{1} \mathrm{H}$ NMR (pyridine- $d_{5}, 600 \mathrm{MHz}$ ) spectrum of the (S)-MTPA ester of 3a..................... S16

Figure S27: ${ }^{1} \mathrm{H}$ NMR (pyridine- $d_{5}, 600 \mathrm{MHz}$ ) spectrum of the (R)-MTPA ester of 3a. ................... S17

Figure S28: ${ }^{1} \mathrm{H}$ NMR (methanol- $d_{4}, 600 \mathrm{MHz}$ ) spectrum of 1a. ..................................................... 17

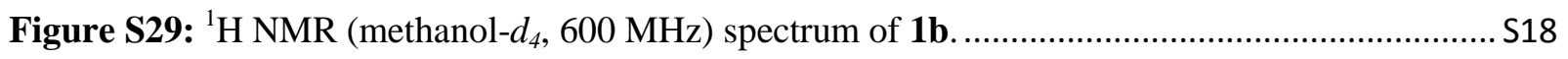

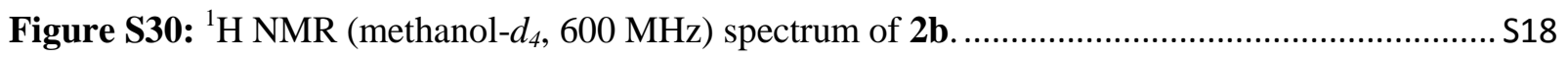




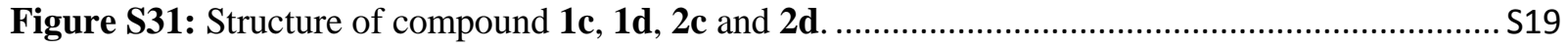

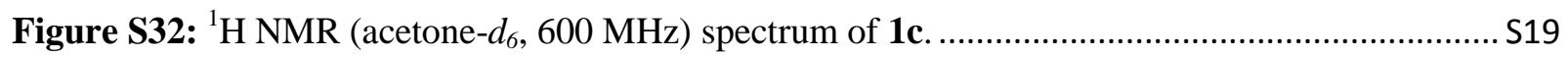

Figure S33: ${ }^{1} \mathrm{H}$ NMR (acetone- $d_{6}, 600 \mathrm{MHz}$ ) spectrum of 1d....................................................... S20

Figure S34: $1 \mathrm{H}$ NMR (methanol- $d_{4}, 600 \mathrm{MHz}$ ) spectrum of 2c................................................... S20

Figure S35: $1 \mathrm{H}$ NMR (methanol- $d_{4}, 600 \mathrm{MHz}$ ) spectrum of 2d. ................................................. S21

Table 1S. ${ }^{1} \mathrm{H}-\mathrm{NMR}$ data for compounds $2 \mathbf{a}$ and $3 \mathbf{a}$ in methanol- $d_{4}, \mathbf{5}$ in DMSO- $d_{6}$ and $\mathbf{6}$ in aceton- $d_{6}$

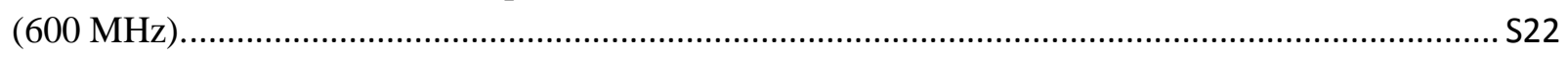

Table 2S. ${ }^{1} \mathrm{H}-\mathrm{NMR}$ data for the S- and R-MTPA monoesters of 1, 2 and 3a (Pyridine- $d_{5}, 30^{\circ} \mathrm{C}$ )...... S23

Table 3S. ${ }^{1} \mathrm{H}-\mathrm{NMR}$ data for compound $\mathbf{1 a}, \mathbf{1 b}, \mathbf{2 b}, \mathbf{2 c}$ and $\mathbf{2 d}$ in methanol- $d_{4}$ and for $\mathbf{1 c}$ and $\mathbf{1 d}$ in

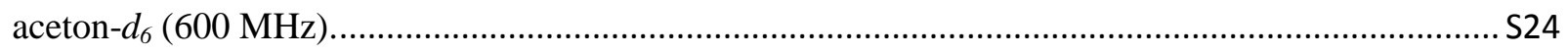




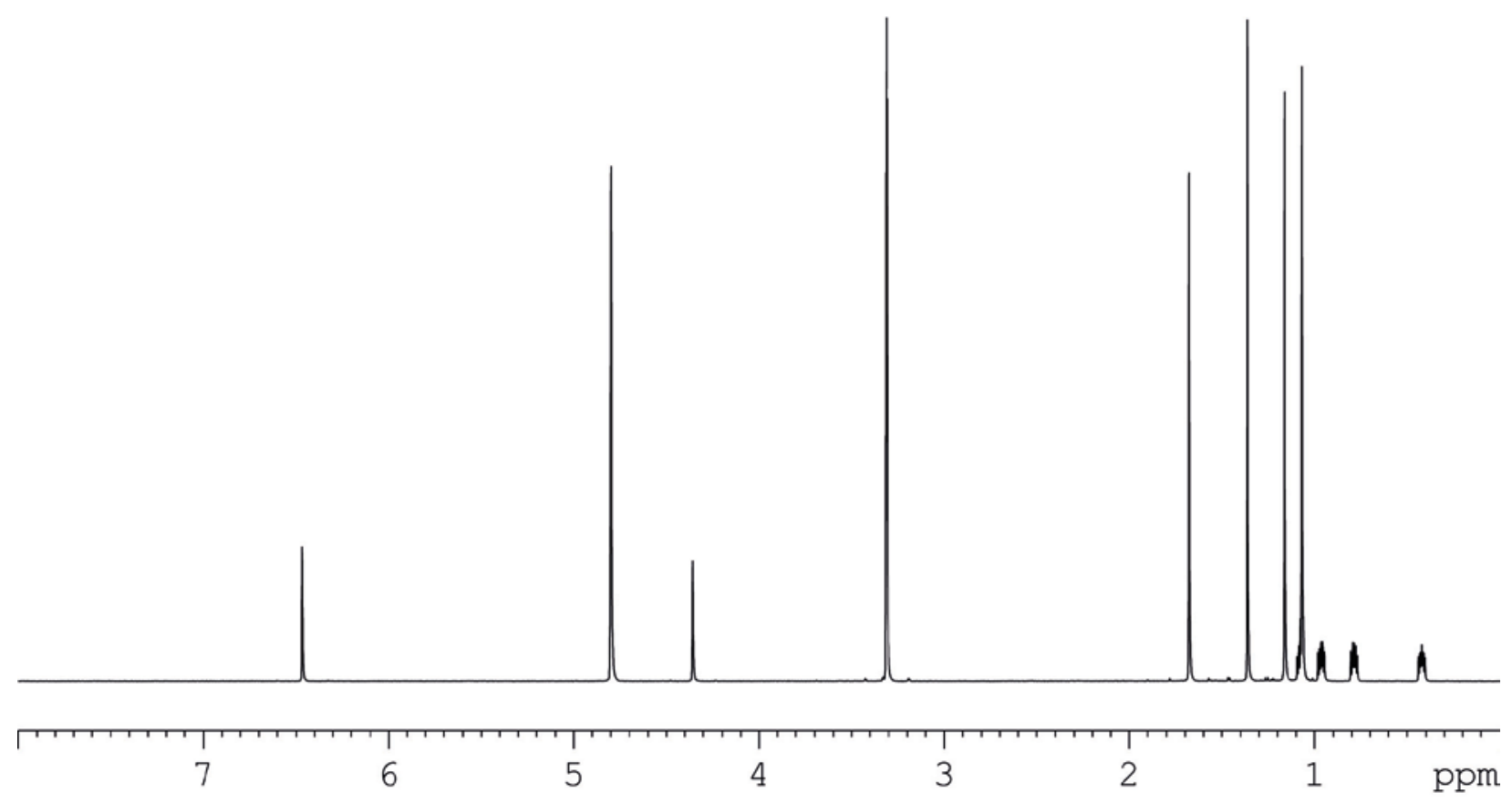

Figure S1: ${ }^{1} \mathrm{H}$ NMR (methanol-d4, $600 \mathrm{MHz}$ ) spectrum of 1.

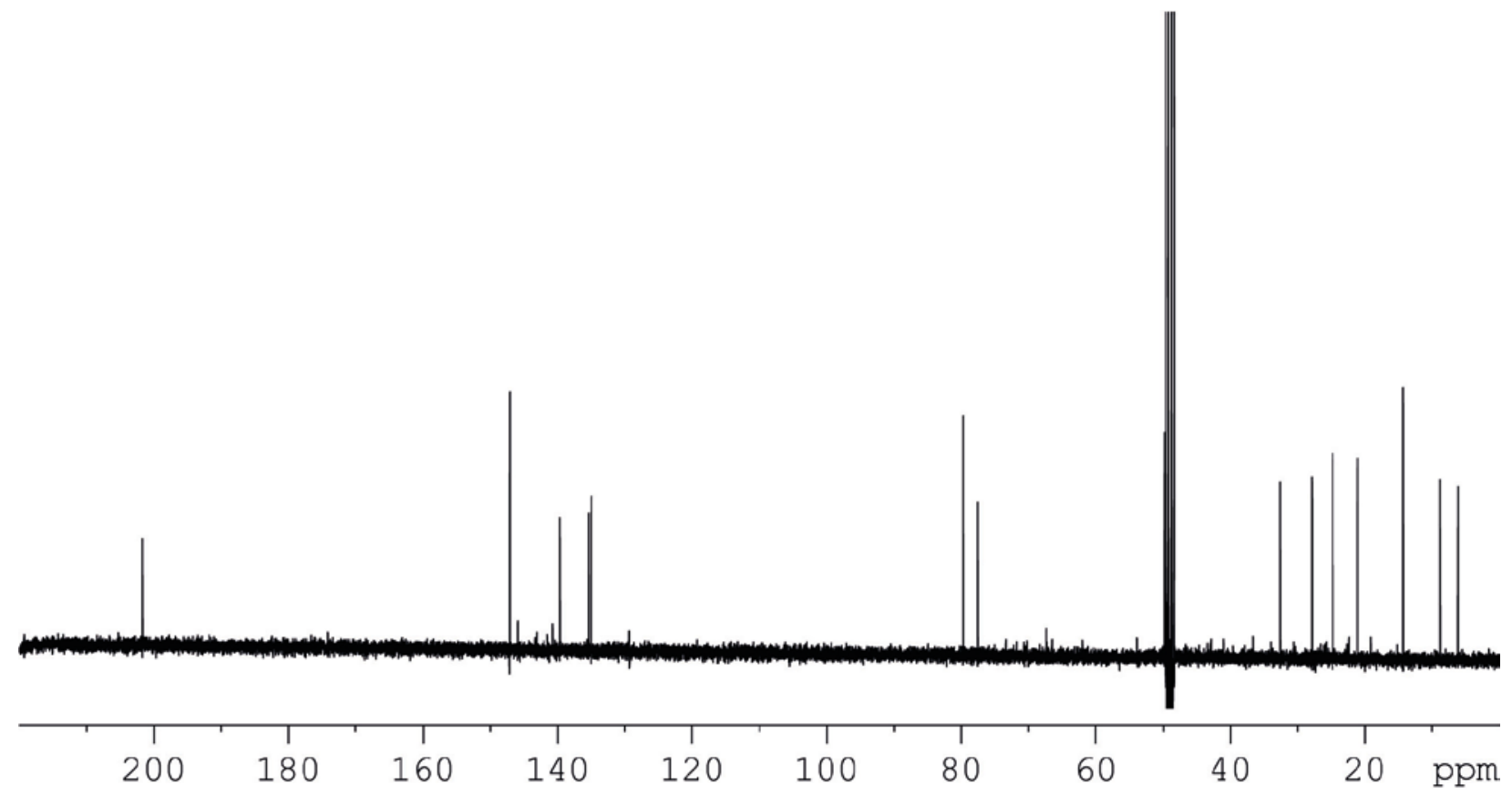

Figure S2: ${ }^{13} \mathrm{C}$ NMR (methanol-d4, $150 \mathrm{MHz}$ ) spectrum of $\mathbf{1}$. 


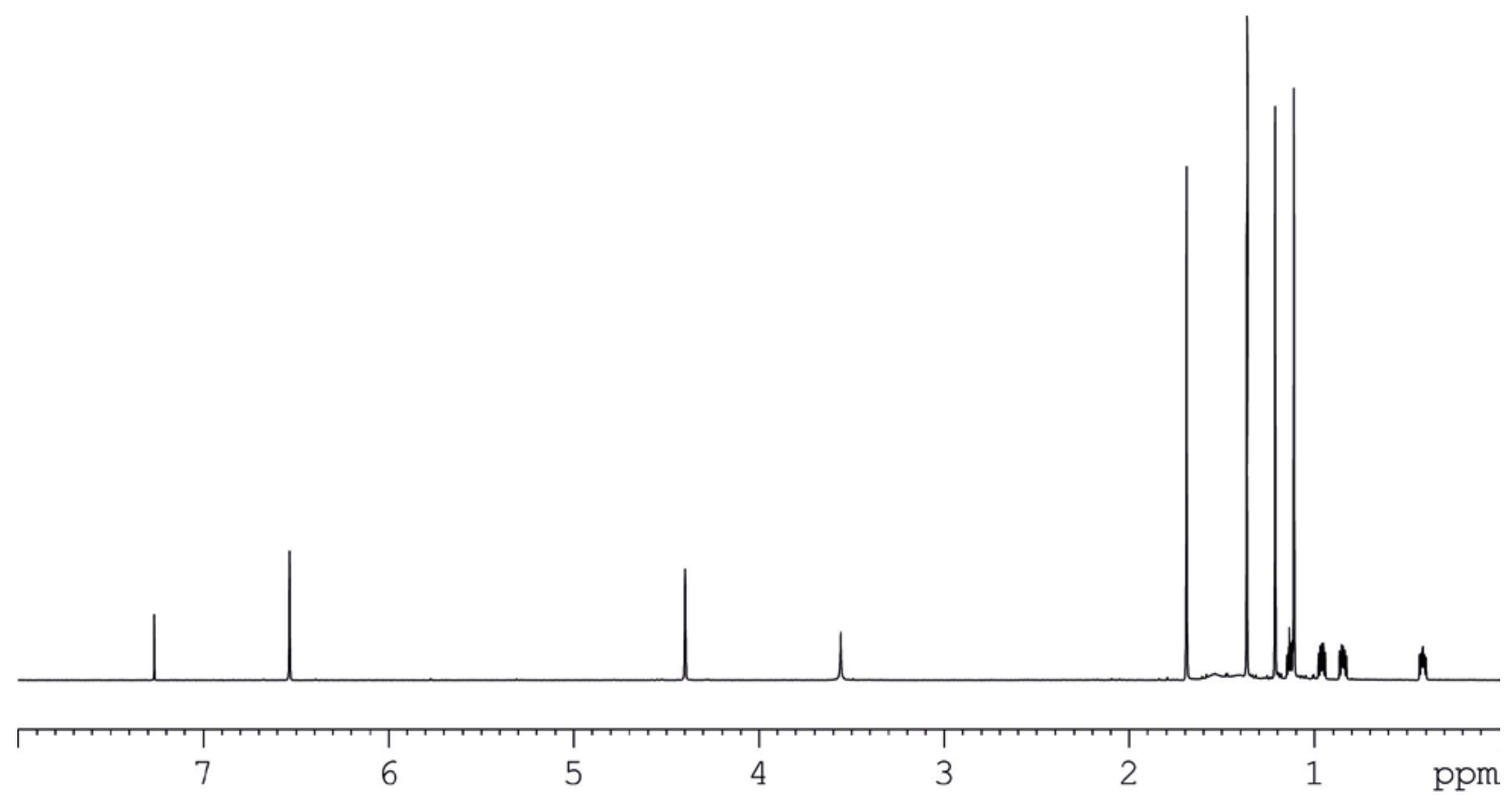

Figure S3: ${ }^{1} \mathrm{H}$ NMR (chloroform-d, $600 \mathrm{MHz}$ ) spectrum of $\mathbf{1}$.

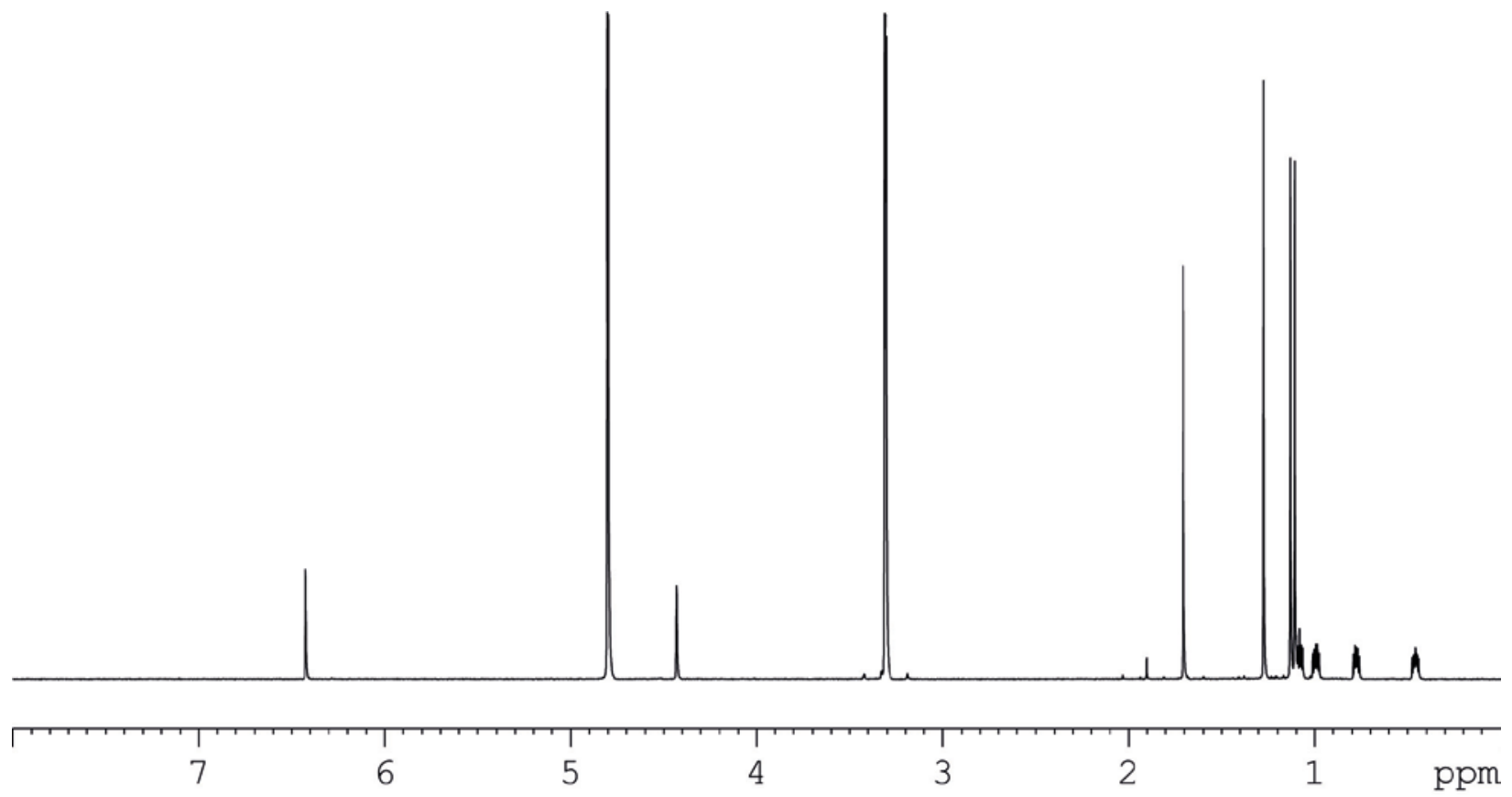

Figure S4: ${ }^{1} \mathrm{H}$ NMR (methanol-d4, $600 \mathrm{MHz}$ ) spectrum of 2. 


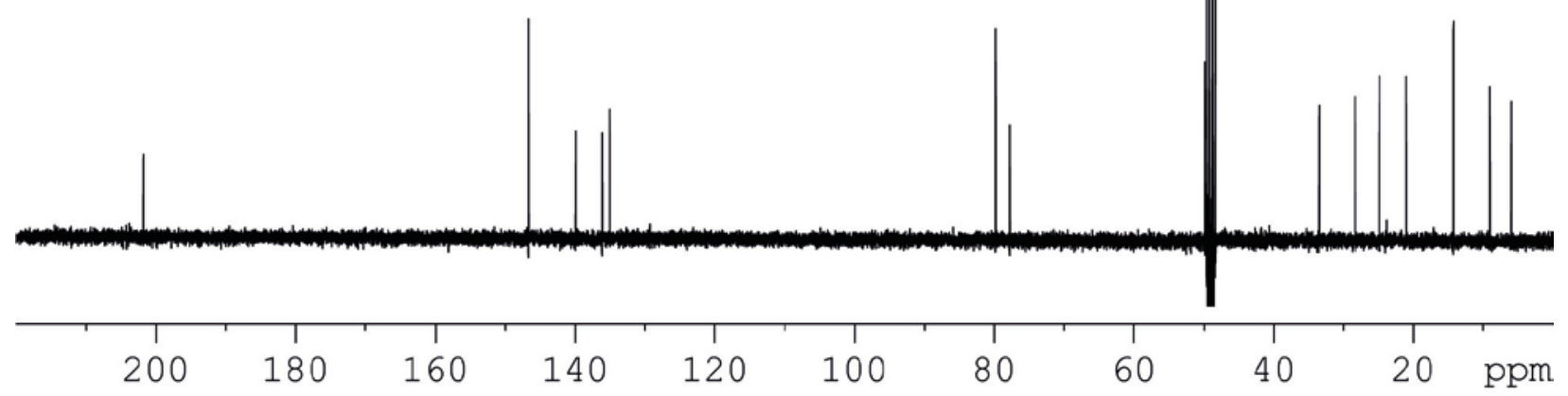

Figure S5: ${ }^{13} \mathrm{C}$ NMR (methanol-d4, $150 \mathrm{MHz}$ ) spectrum of 2.

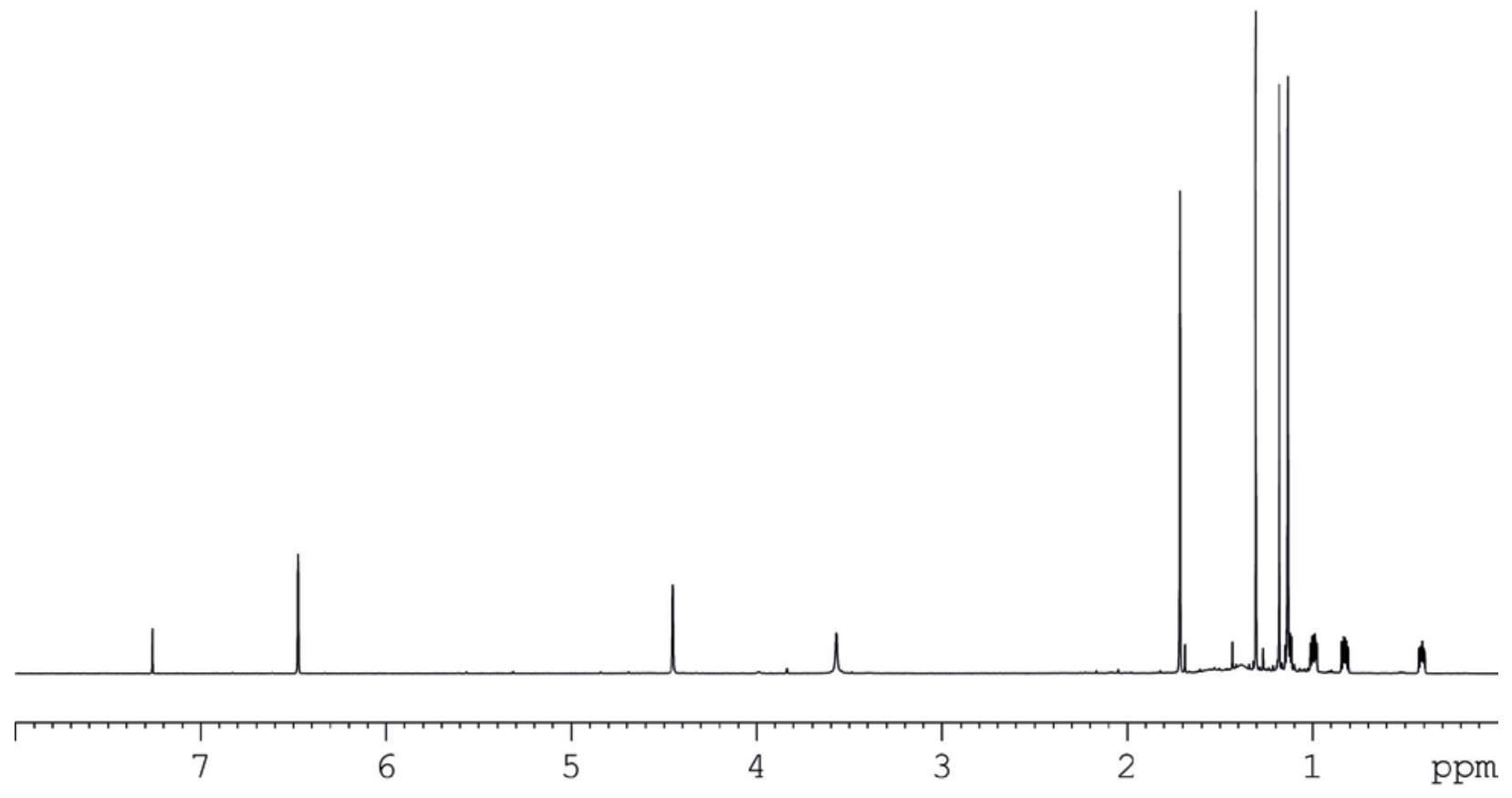

Figure S6: ${ }^{1} \mathrm{H}$ NMR (chloroform-d, $600 \mathrm{MHz}$ ) spectrum of 2. 


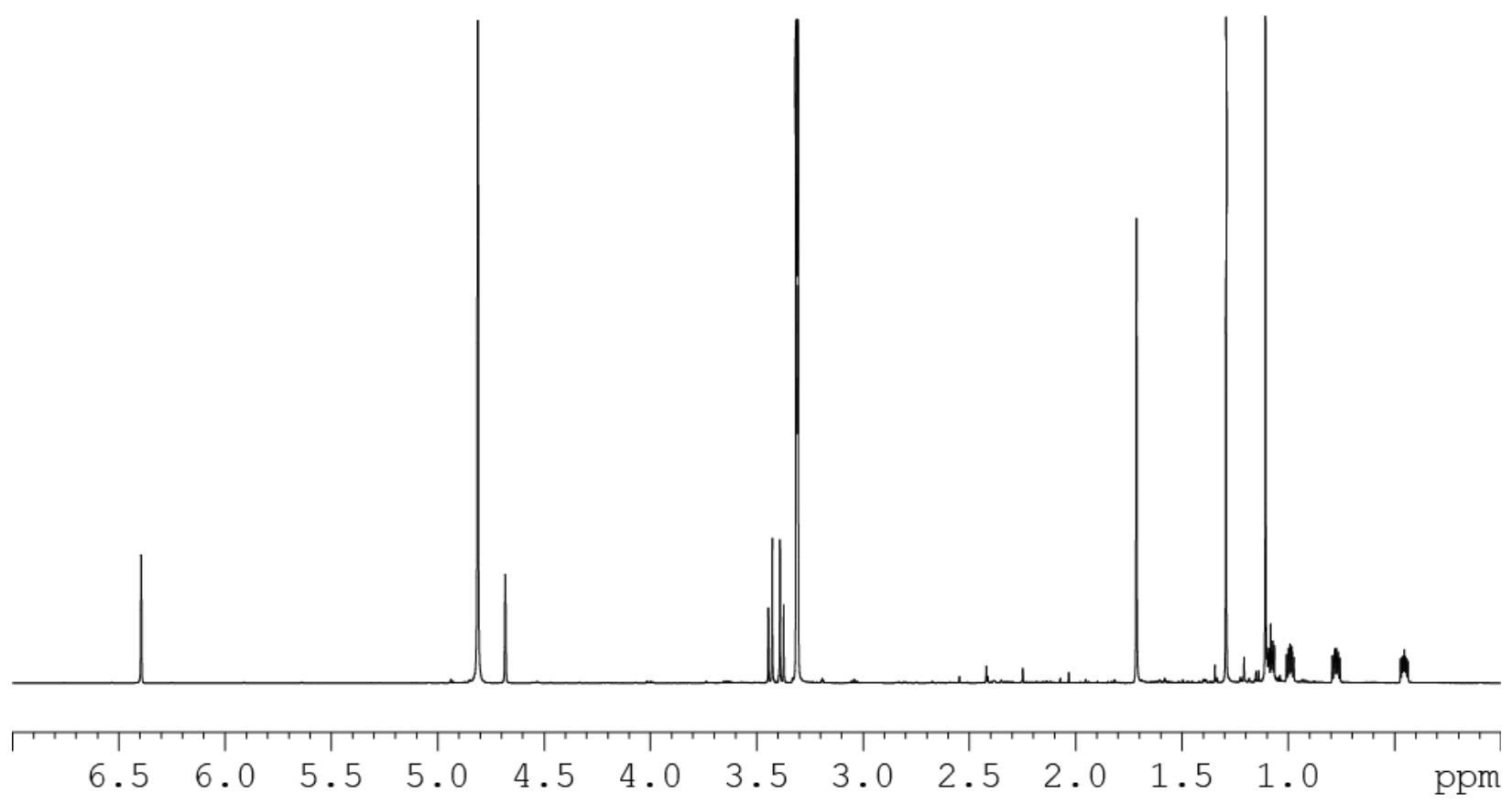

Figure S7: ${ }^{1} \mathrm{H}$ NMR (methanol-d4, $600 \mathrm{MHz}$ ) spectrum of 3.

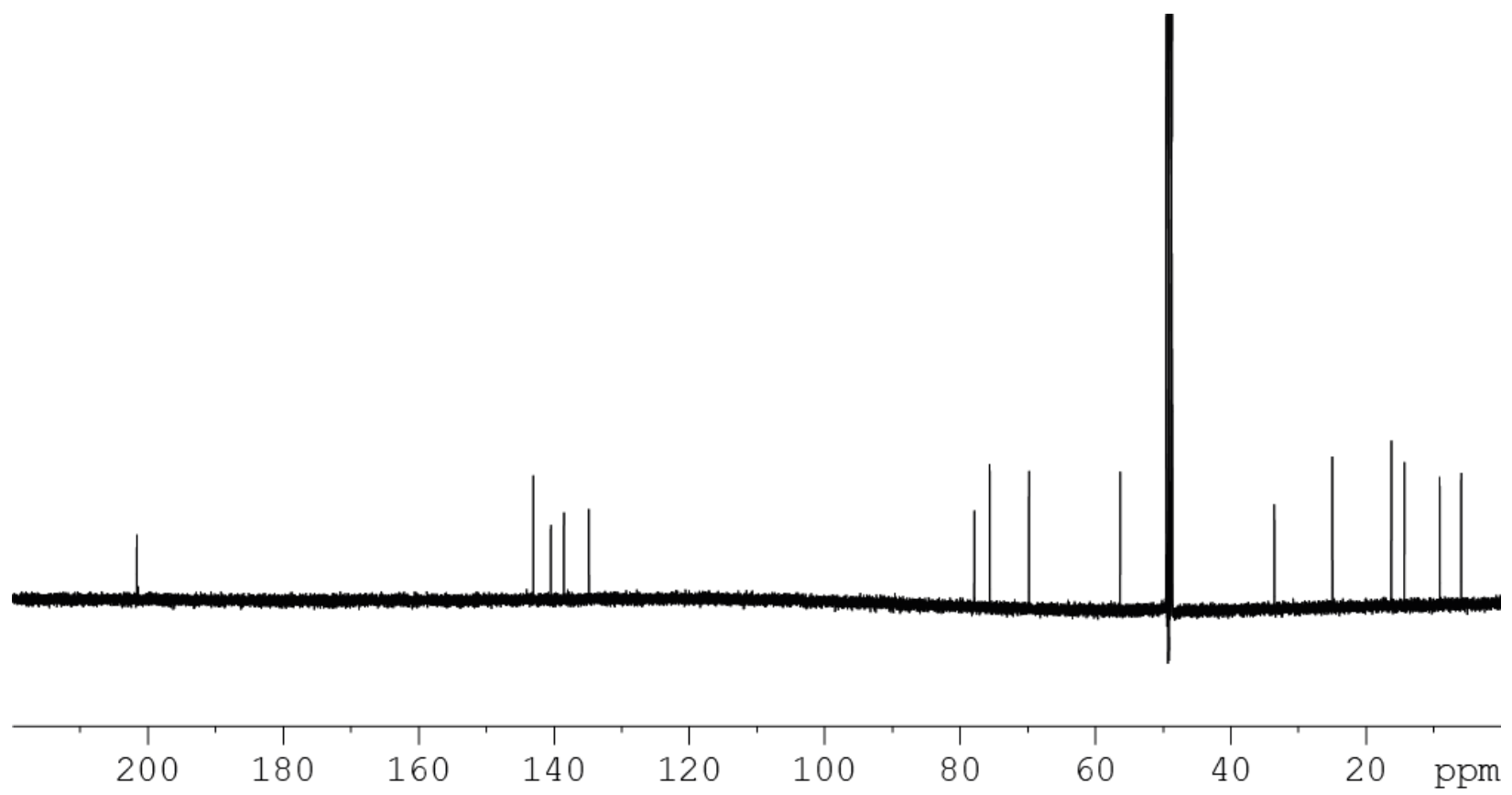

Figure S8: ${ }^{13} \mathrm{C}$ NMR (methanol-d4, $150 \mathrm{MHz}$ ) spectrum of 3. 


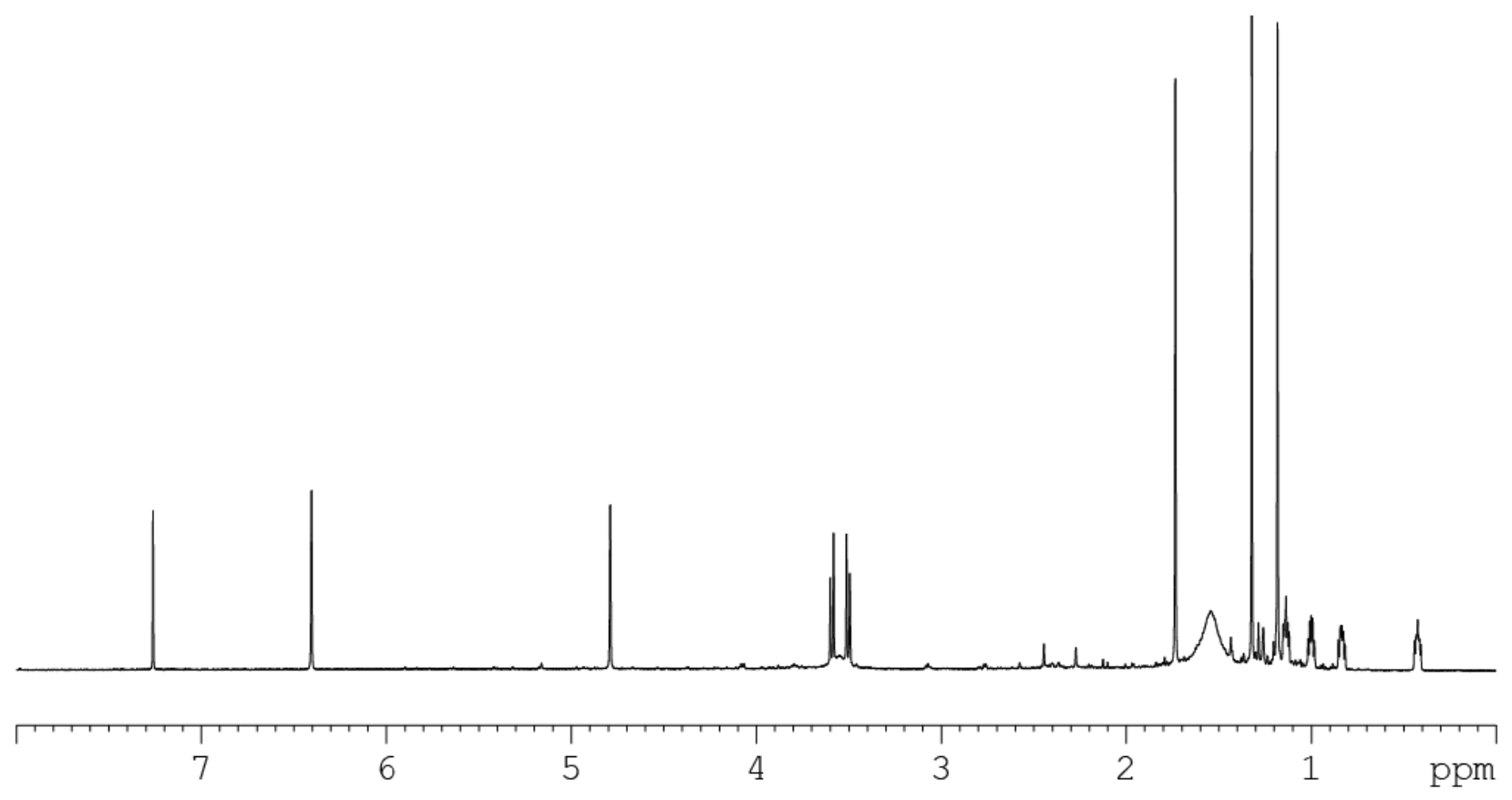

Figure S9: ${ }^{1} \mathrm{H}$ NMR (chloroform-d, $600 \mathrm{MHz}$ ) spectrum of 3.

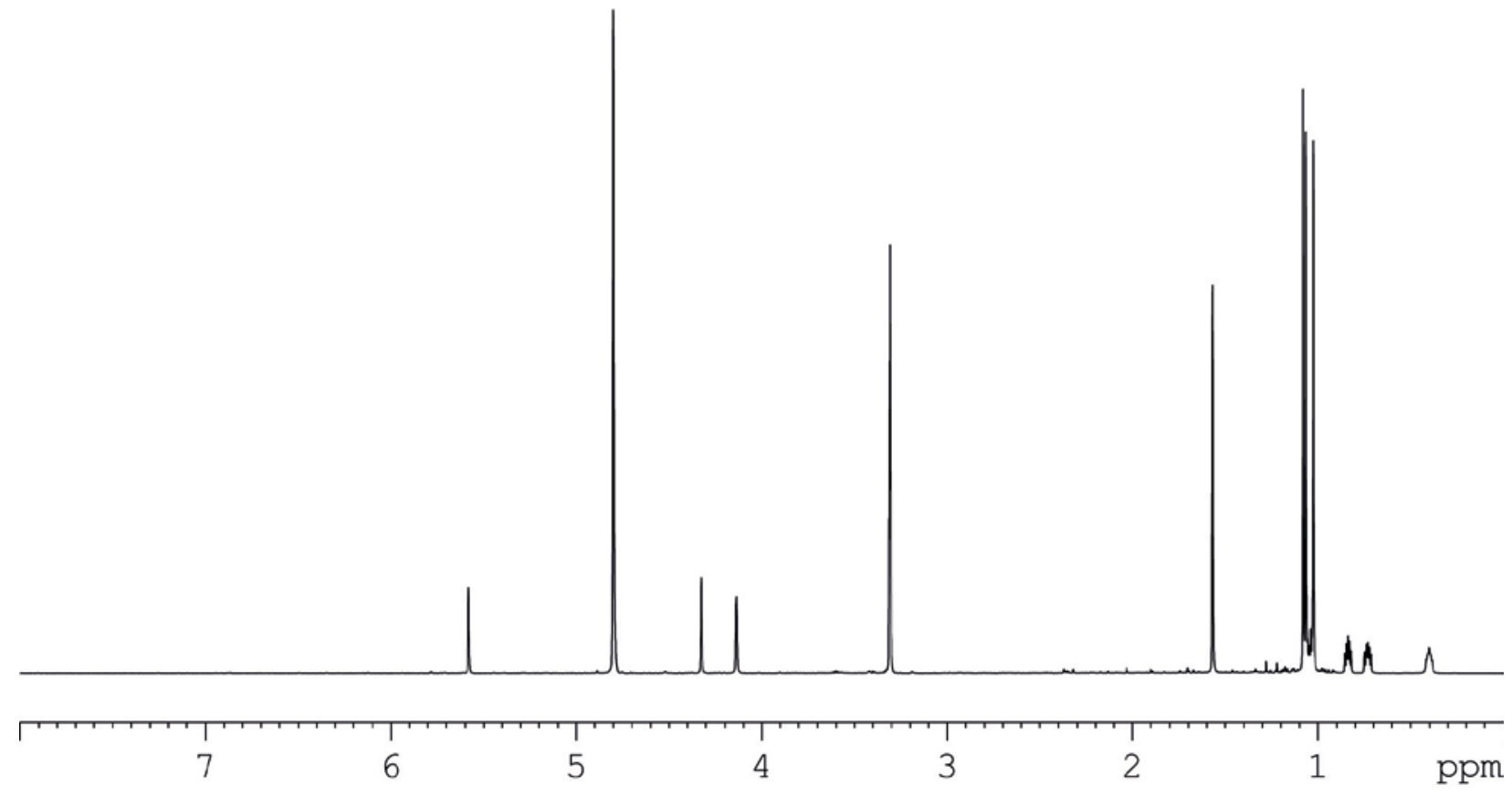

Figure S10: ${ }^{1} \mathrm{H}$ NMR (methanol-d4, $600 \mathrm{MHz}$ ) spectrum of 4 . 


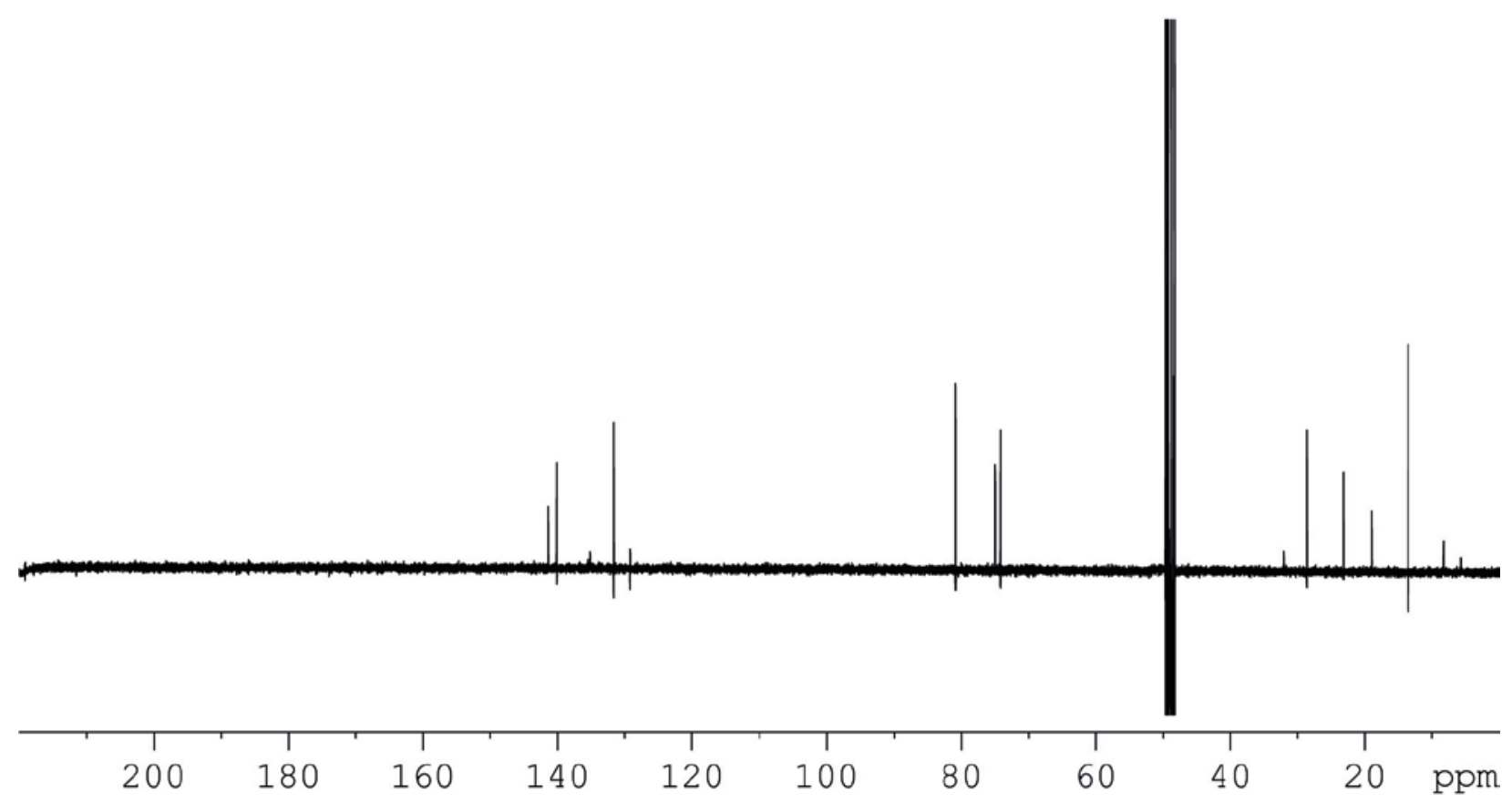

Figure S11: ${ }^{13} \mathrm{C}$ NMR (methanol-d4, $150 \mathrm{MHz}$ ) spectrum of 4.

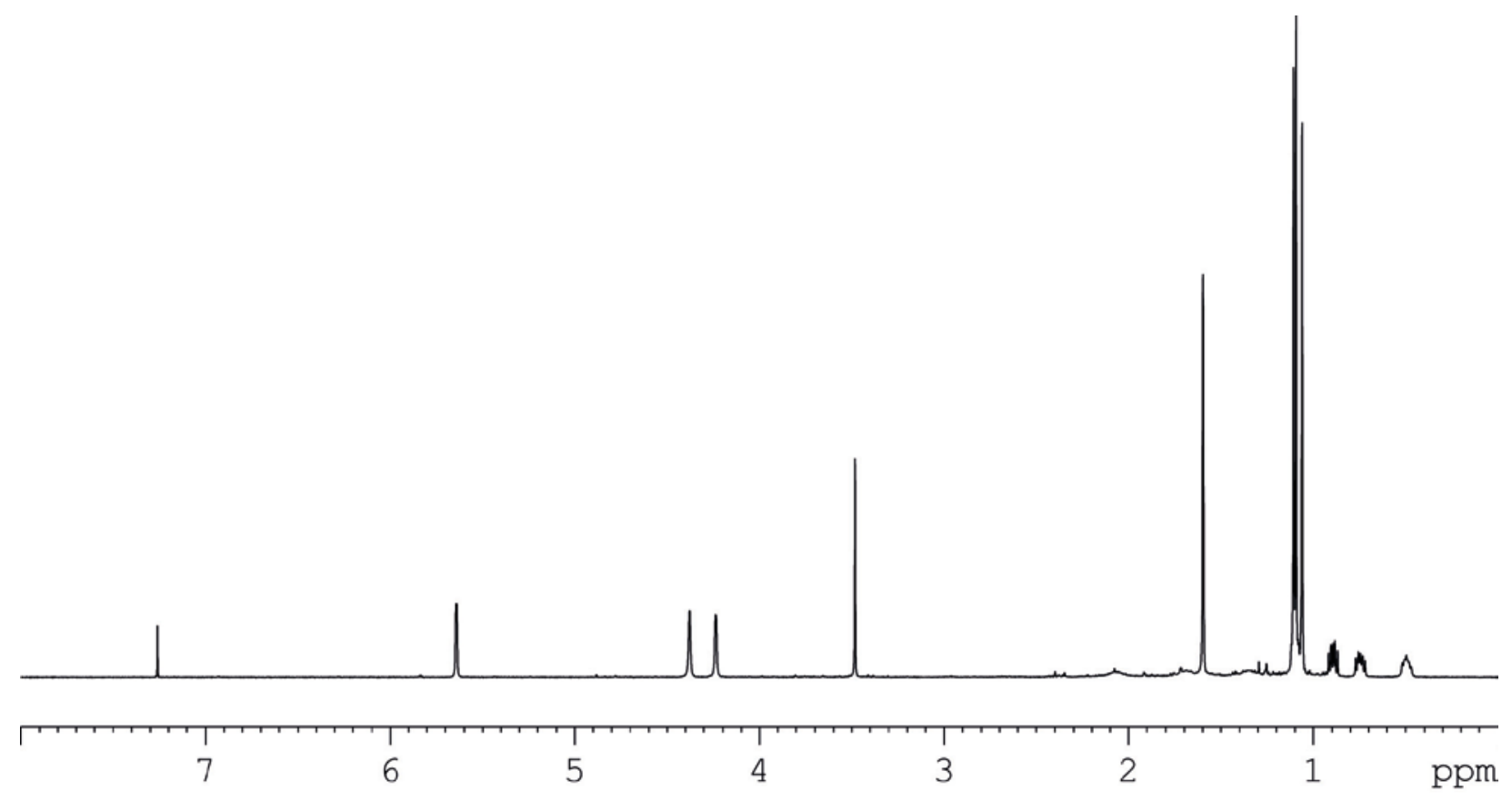

Figure S12: ${ }^{1} \mathrm{H}$ NMR (chloroform-d, $600 \mathrm{MHz}$ ) spectrum of 4. 


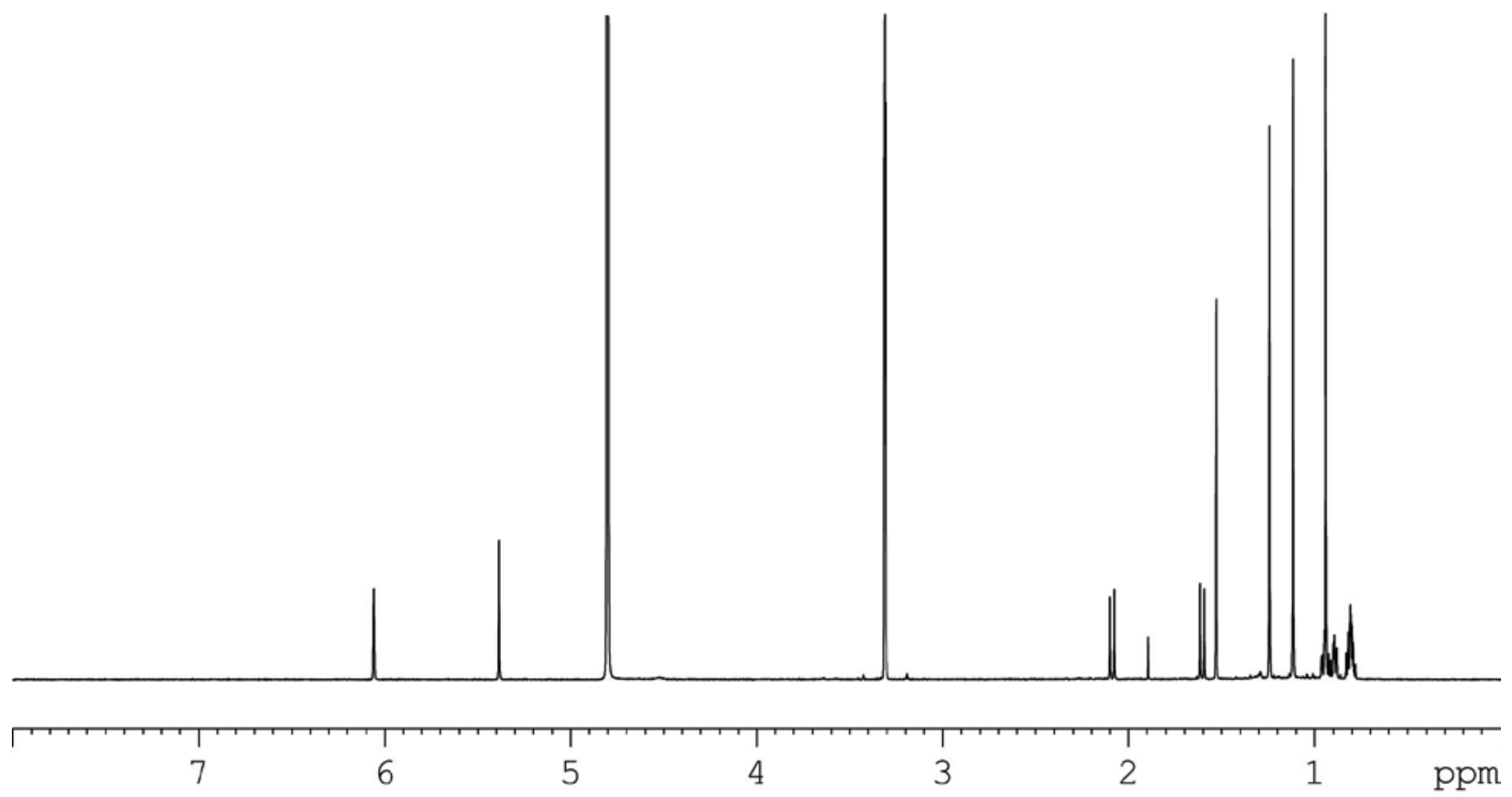

Figure S13: ${ }^{1} \mathrm{H}$ NMR (methanol-d4, $600 \mathrm{MHz}$ ) spectrum of 5.

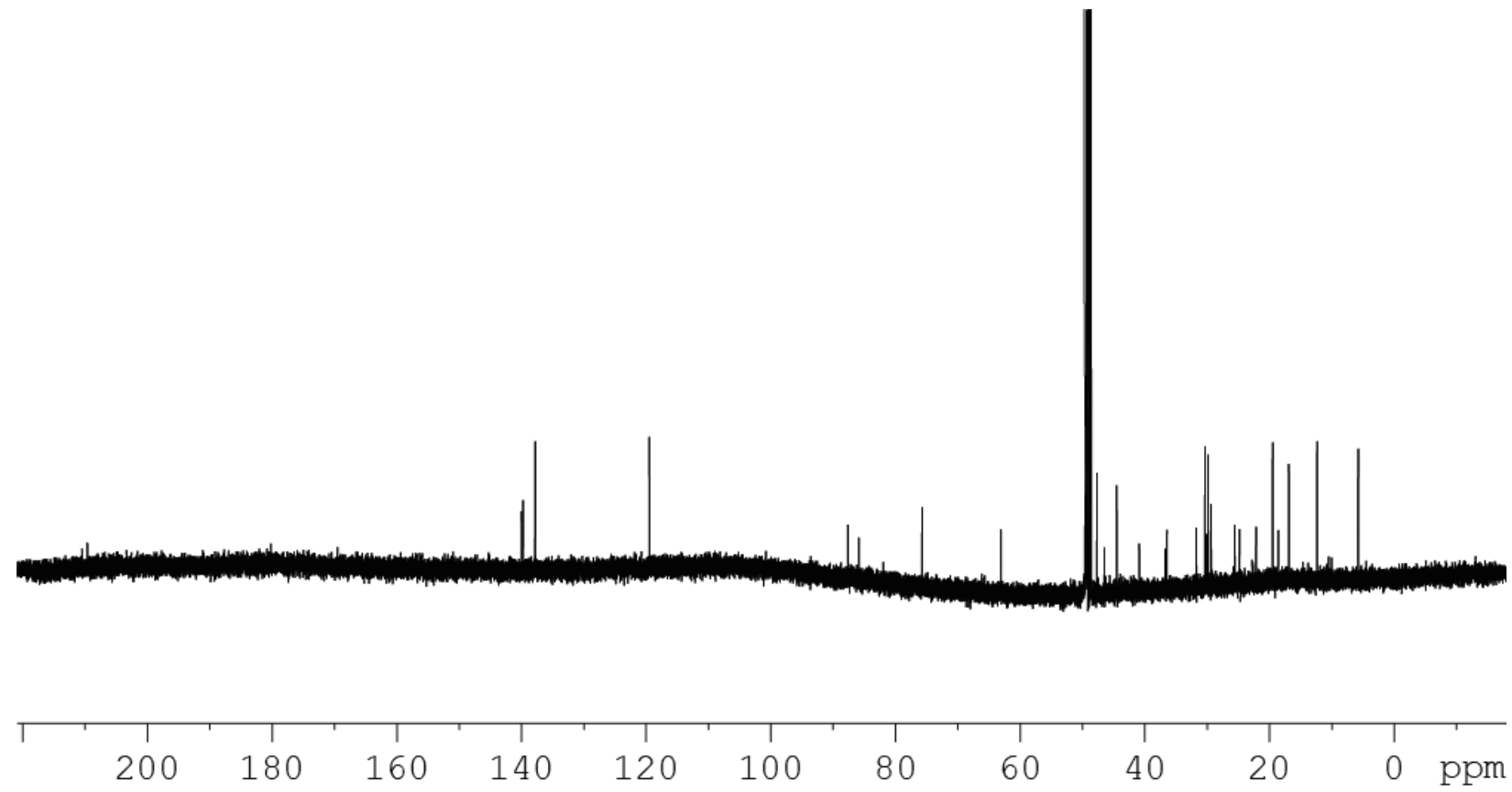

Figure S14: ${ }^{13} \mathrm{C}$ NMR (methanol-d4, $150 \mathrm{MHz}$ ) spectrum of 5. 


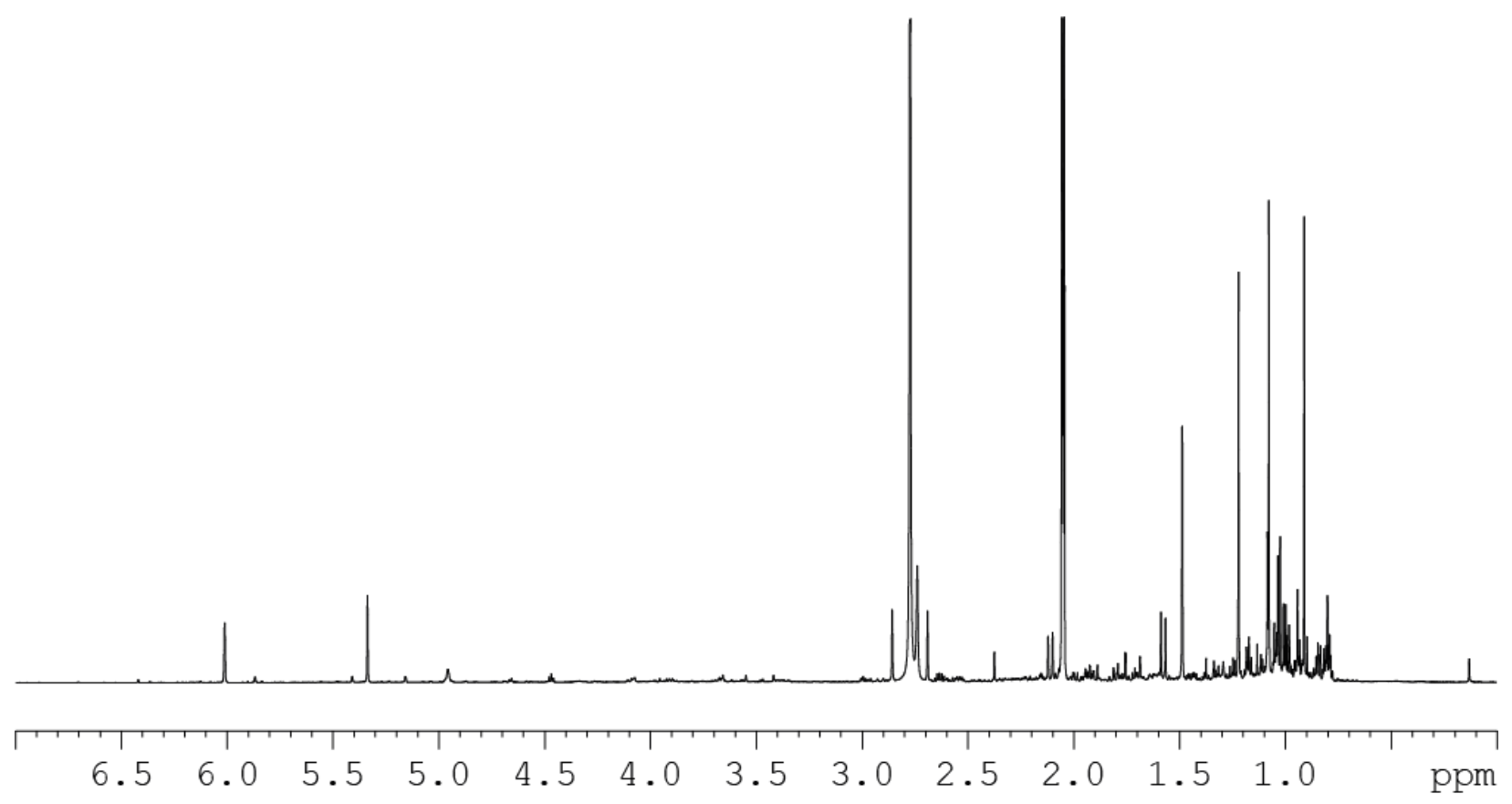

Figure S15: ${ }^{1} \mathrm{H}$ NMR (acetone-d6, $600 \mathrm{MHz}$ ) spectrum of 5.

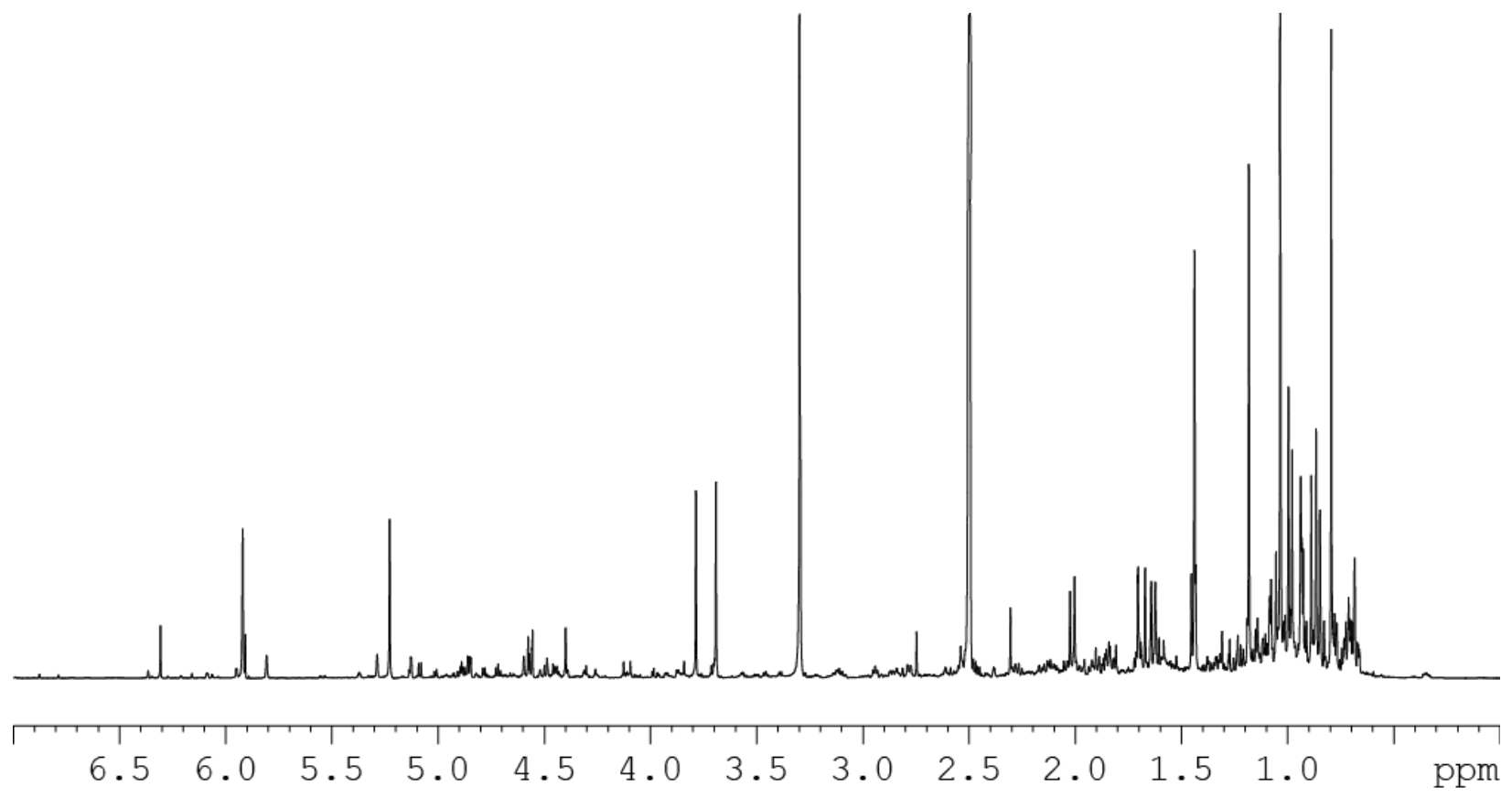

Figure S16: ${ }^{1} \mathrm{H}$ NMR (DMSO-d6, $600 \mathrm{MHz}$ ) spectrum of 5. 


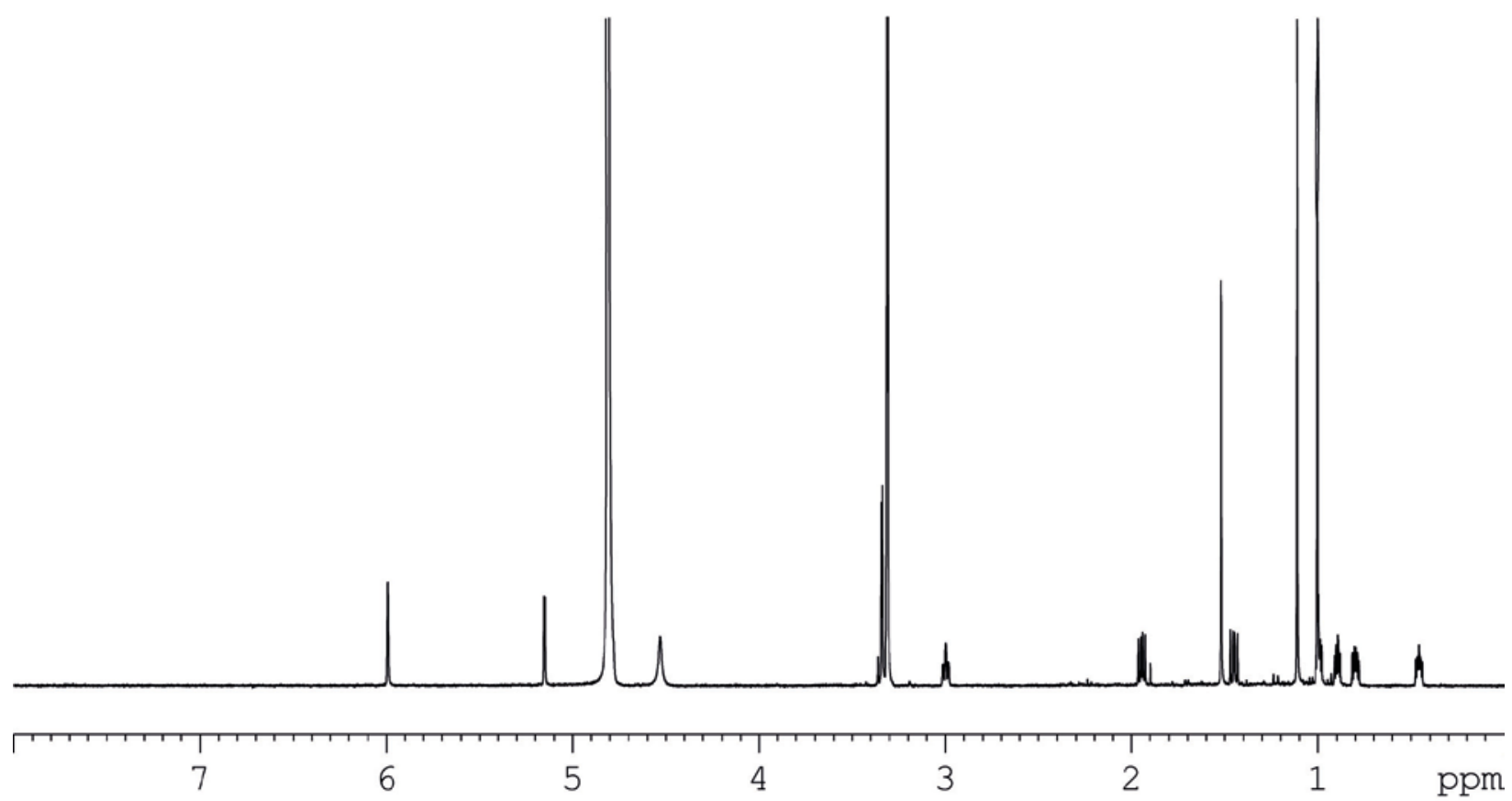

Figure S17: ${ }^{1} \mathrm{H}$ NMR (methanol-d4, $600 \mathrm{MHz}$ ) spectrum of 6.

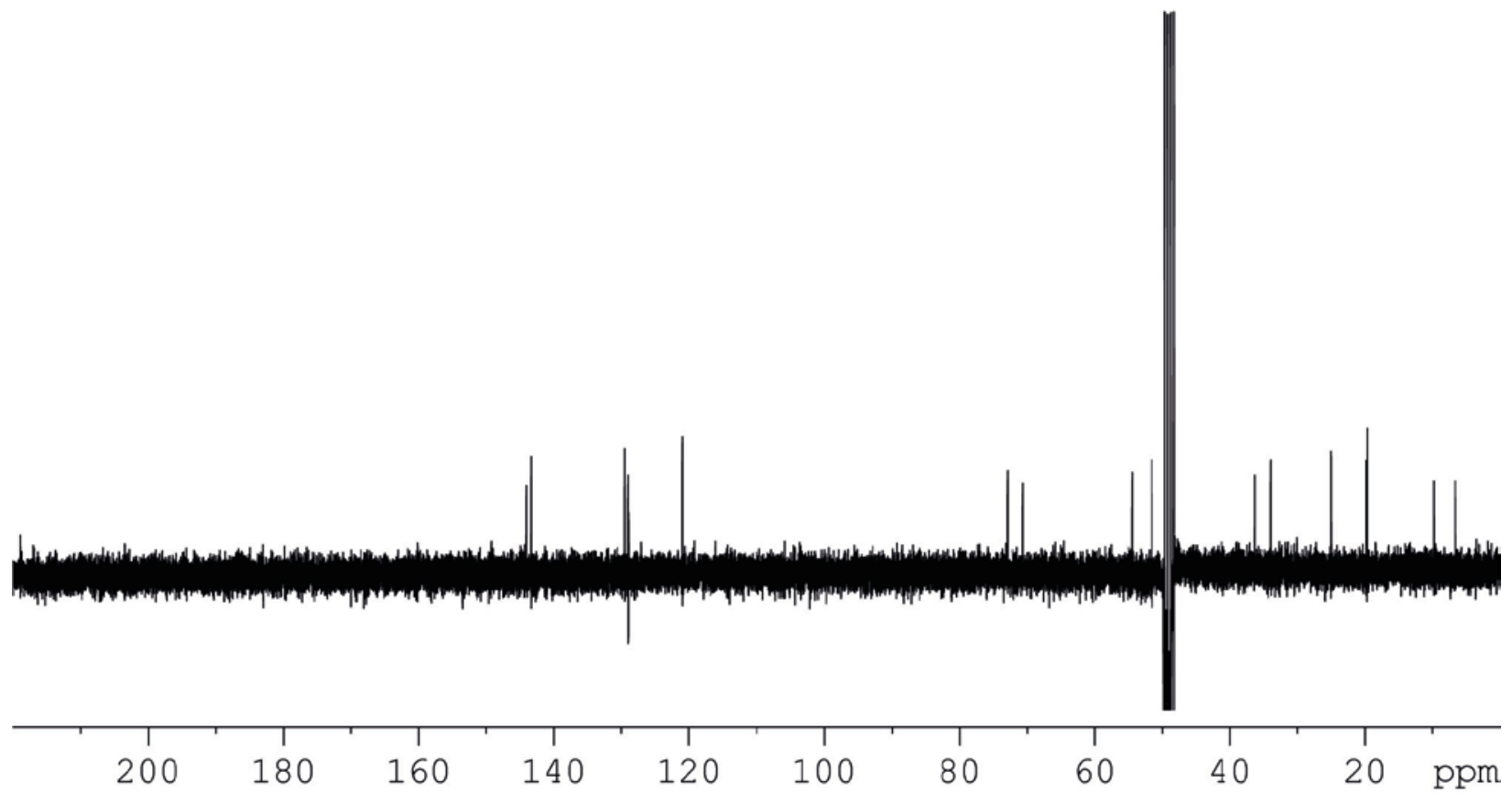

Figure S18: ${ }^{13} \mathrm{C}$ NMR (methanol-d4, $150 \mathrm{MHz}$ ) spectrum of $\mathbf{6}$. 


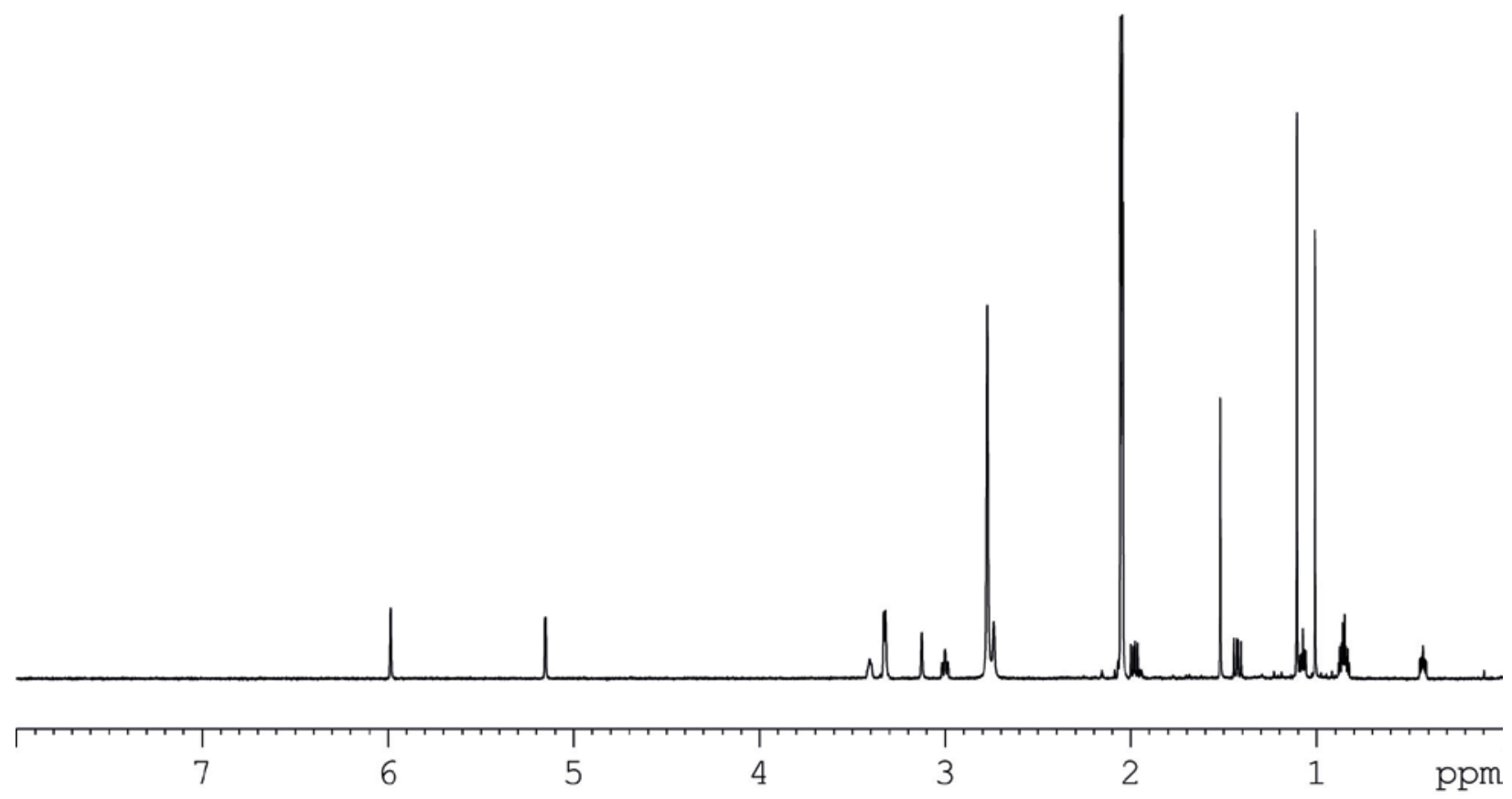

Figure S19: ${ }^{1} \mathrm{H}$ NMR (acetone-d6, $600 \mathrm{MHz}$ ) spectrum of 6.

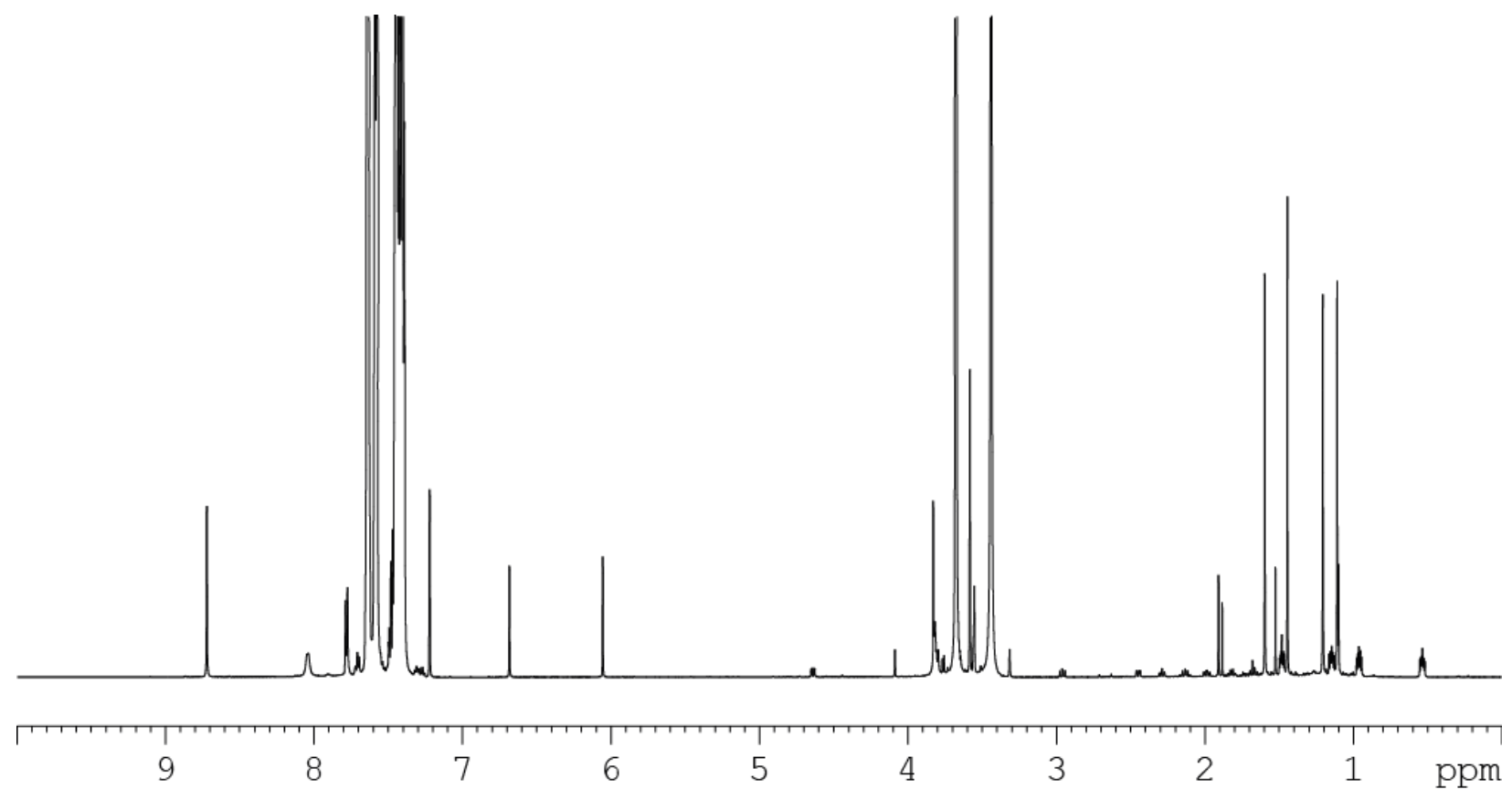

Figure S20: ${ }^{1} \mathrm{H}$ NMR (pyridine-d5, $600 \mathrm{MHz}$ ) spectrum of the (S)-MTPA ester of $\mathbf{1}$. 


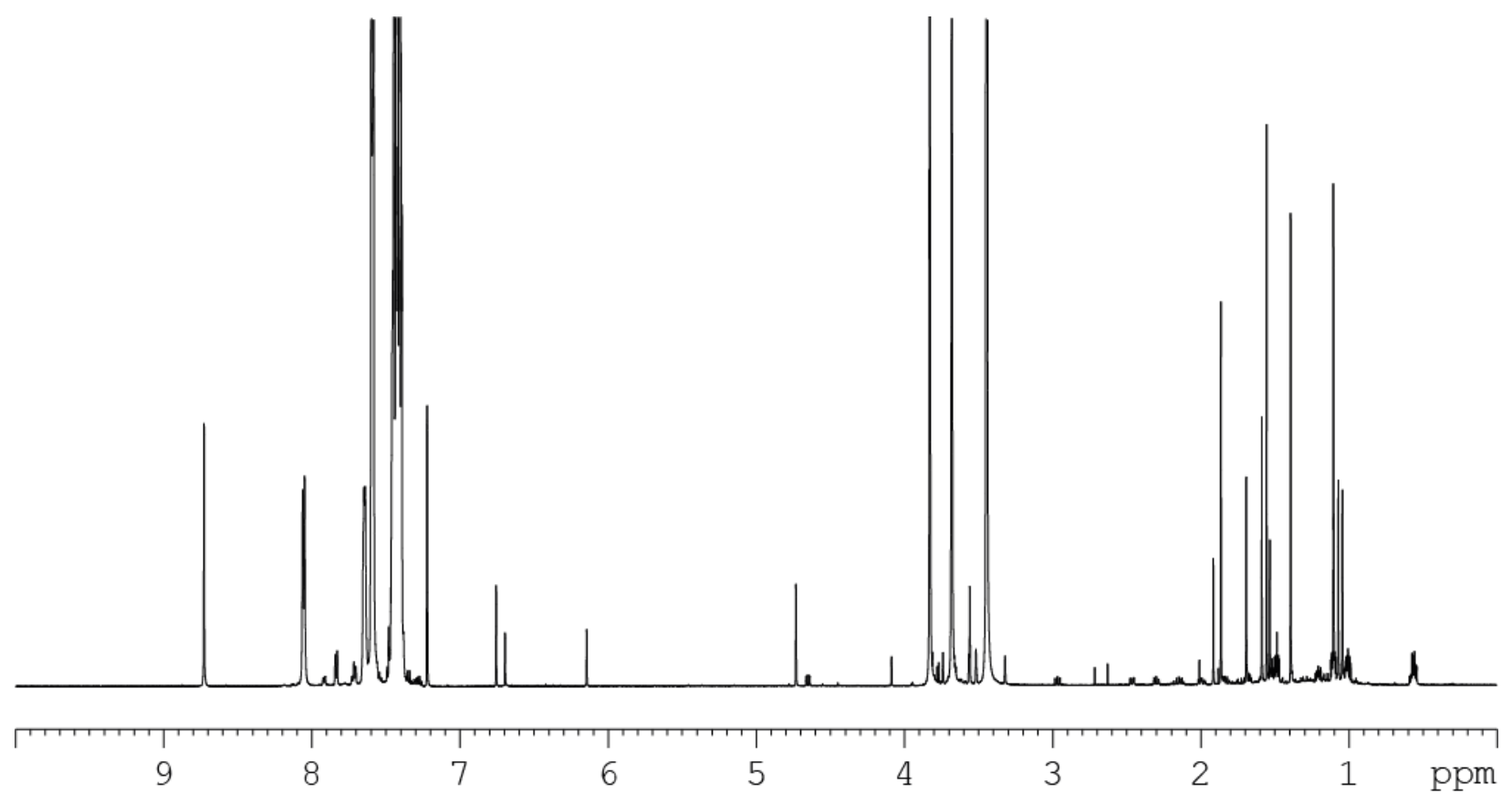

Figure S21: ${ }^{1} \mathrm{H}$ NMR (pyridine-d5, $600 \mathrm{MHz}$ ) spectrum of the (R)-MTPA ester of 1.

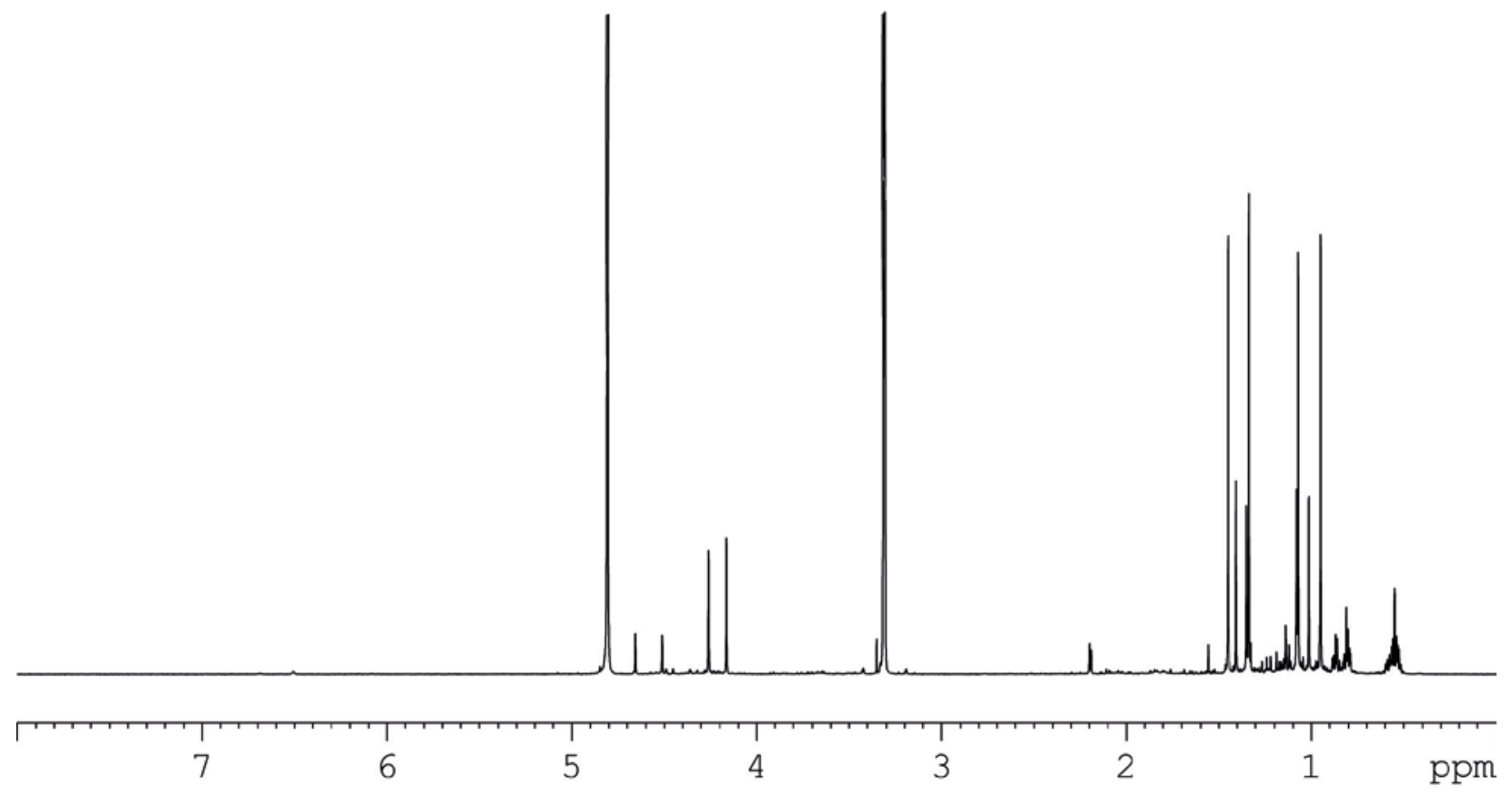

Figure S22: ${ }^{1} \mathrm{H}$ NMR (methanol-d4, $600 \mathrm{MHz}$ ) spectrum of 2a. 


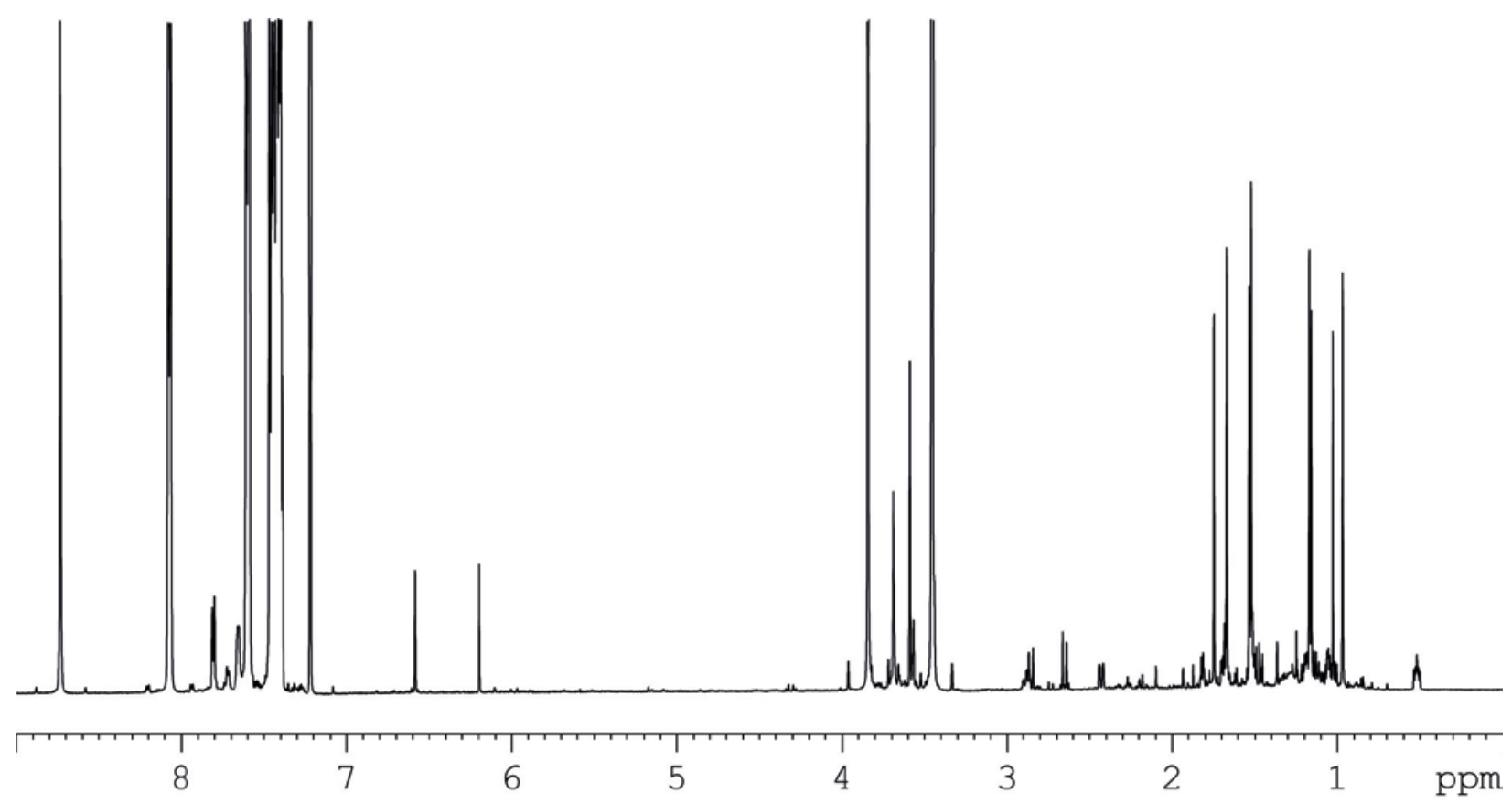

Figure S23: ${ }^{1} \mathrm{H}$ NMR (pyridine-d5, $600 \mathrm{MHz}$ ) spectrum of the (S)-MTPA ester of 2.

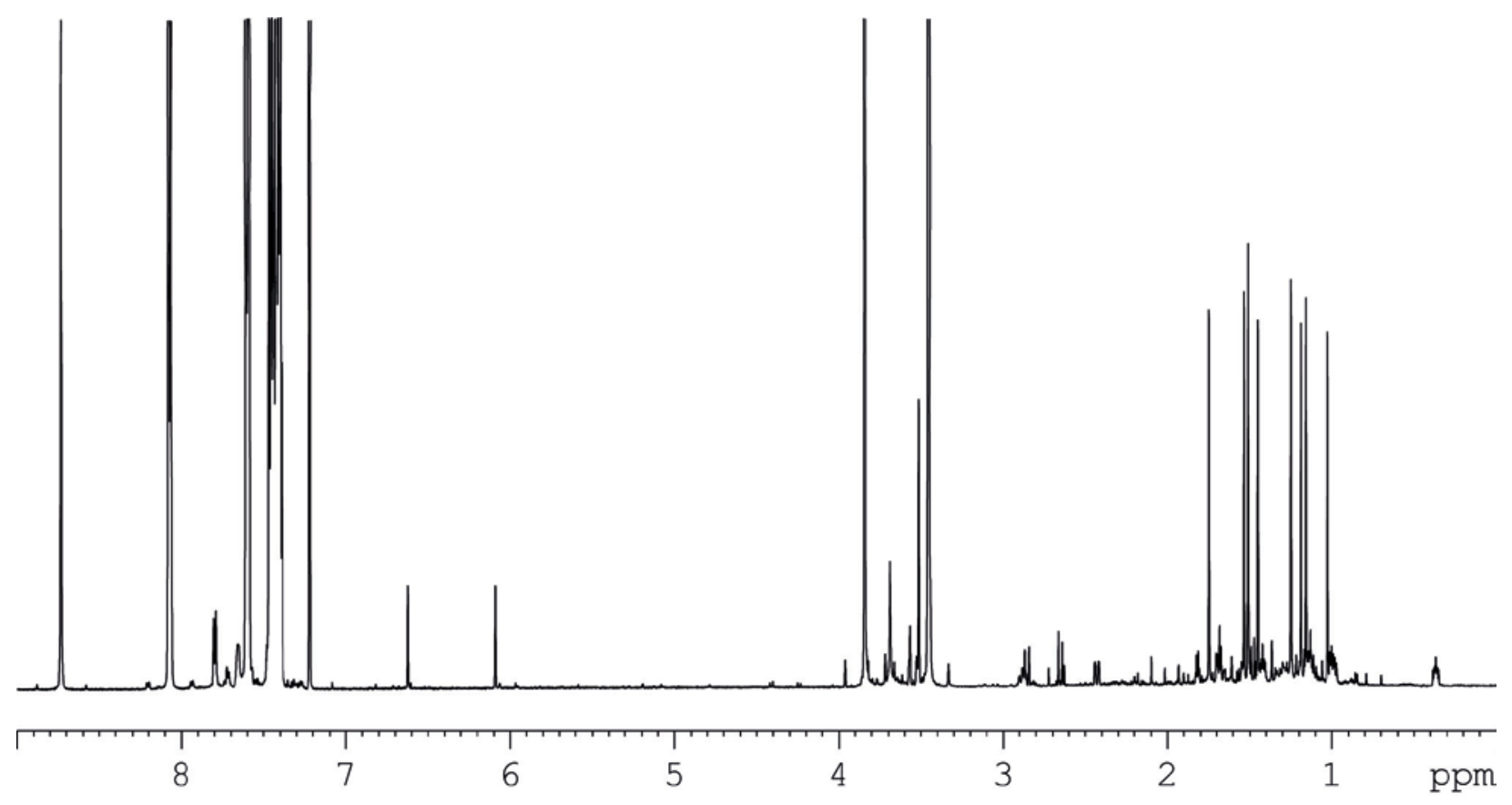

Figure S24: ${ }^{1} \mathrm{H}$ NMR (pyridine- $d 5,600 \mathrm{MHz}$ ) spectrum of the (R)-MTPA ester of 2. 


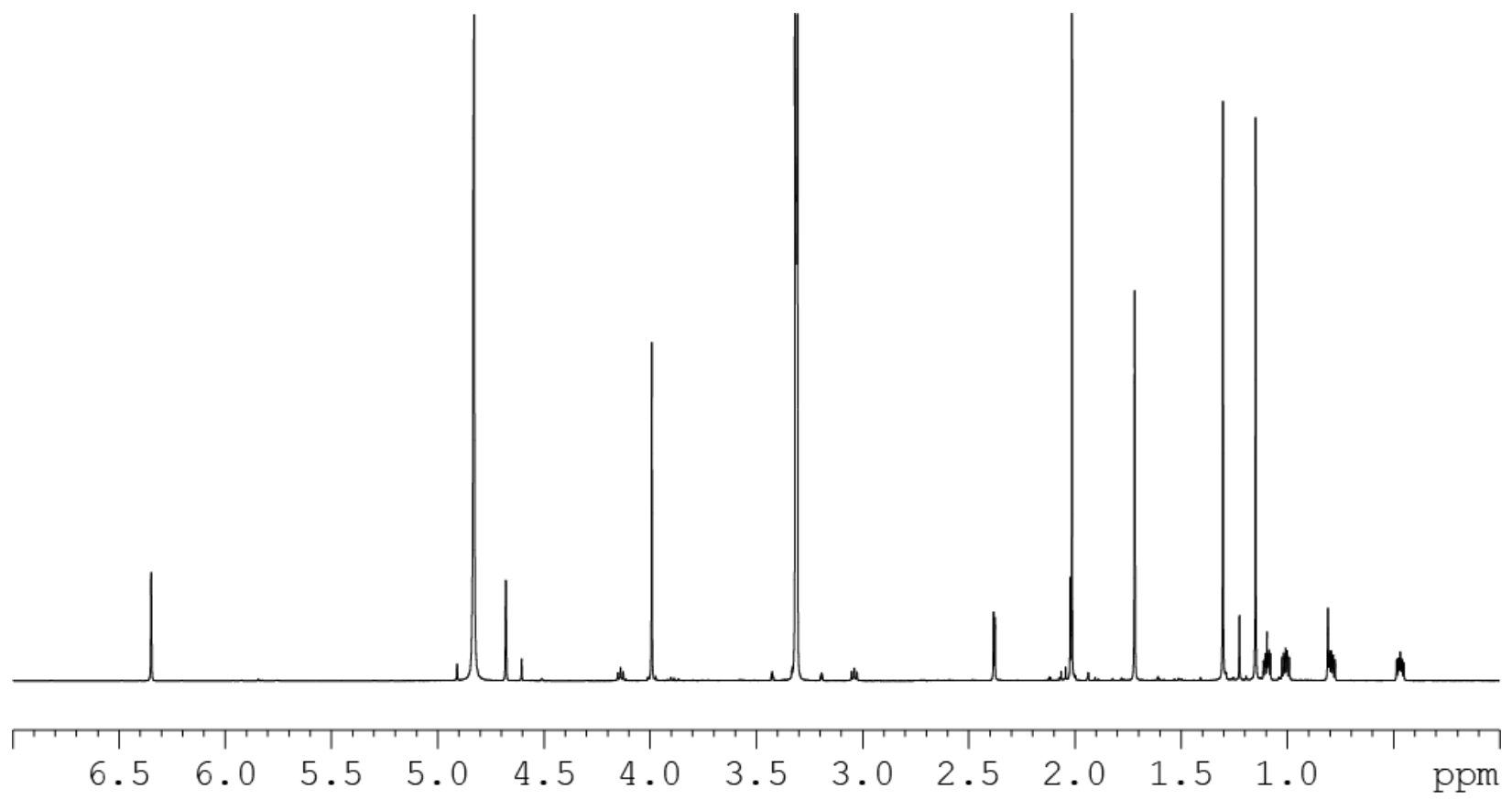

Figure S25: ${ }^{1} \mathrm{H}$ NMR (methanol-d4, $600 \mathrm{MHz}$ ) spectrum of 3a.

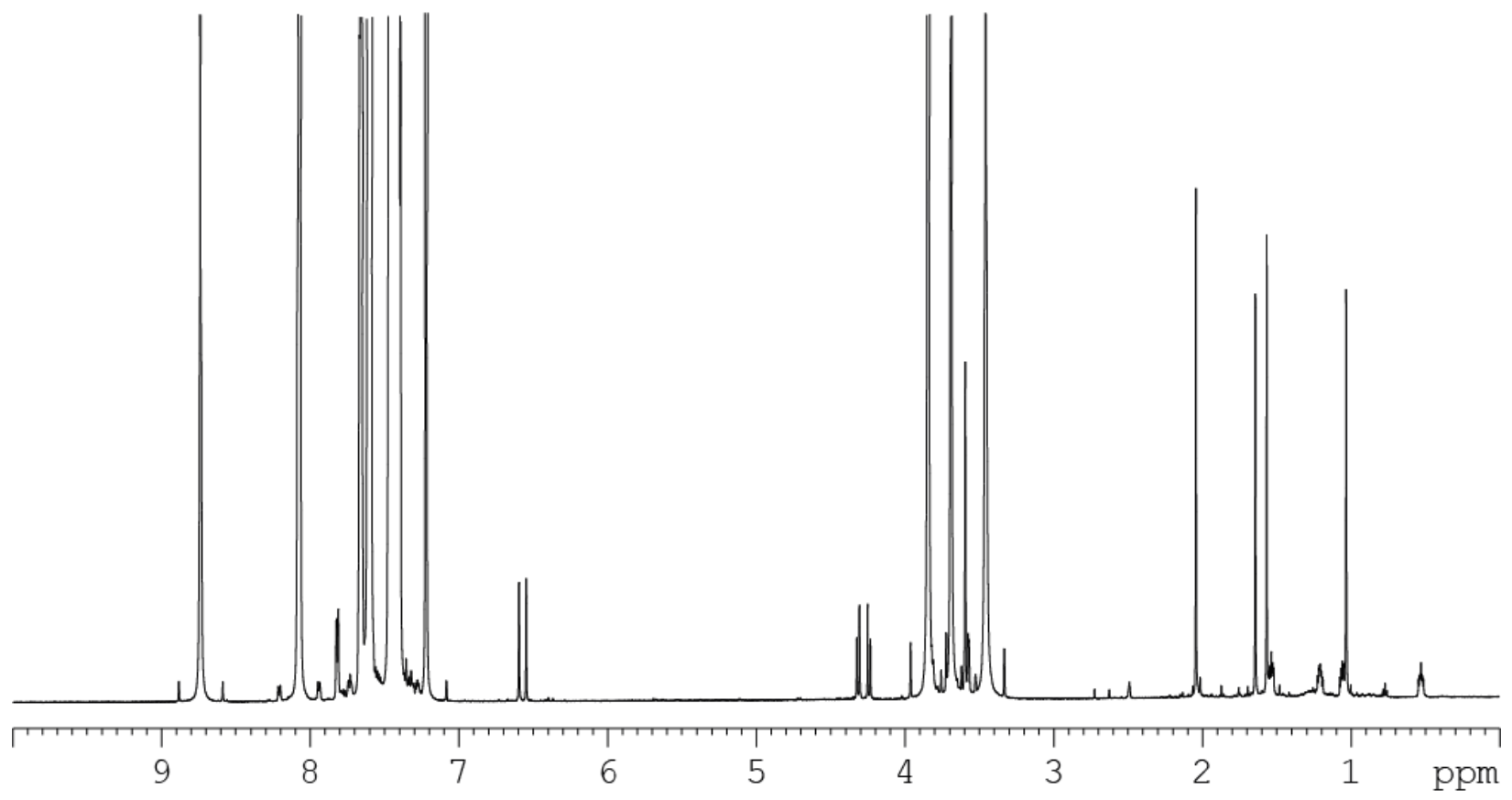

Figure S26: ${ }^{1} \mathrm{H}$ NMR (pyridine- $d_{5}, 600 \mathrm{MHz}$ ) spectrum of the (S)-MTPA ester of 3a. 


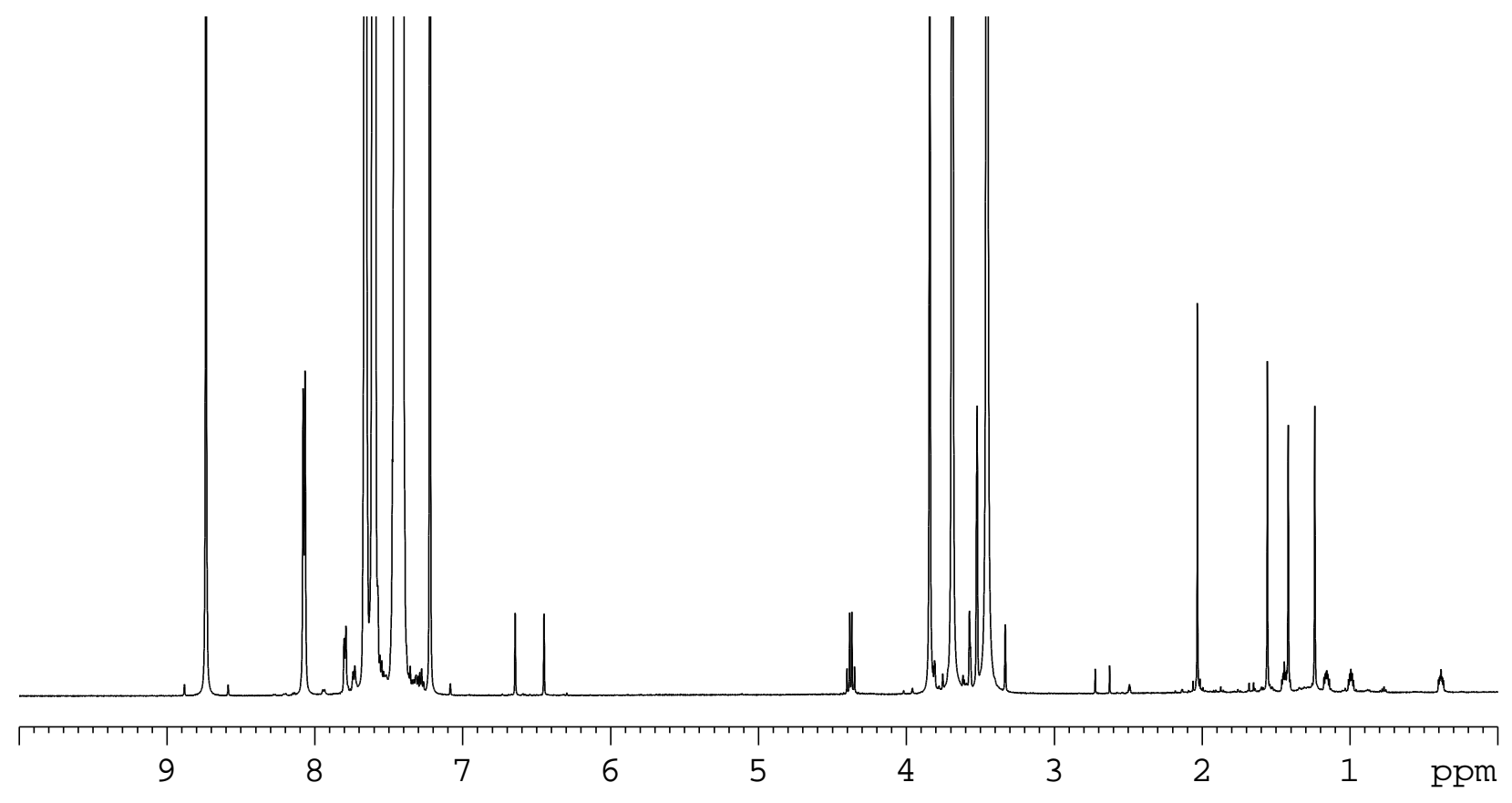

Figure S27: ${ }^{1} \mathrm{H}$ NMR (pyridine- $d_{5}, 600 \mathrm{MHz}$ ) spectrum of the (R)-MTPA ester of 3a.

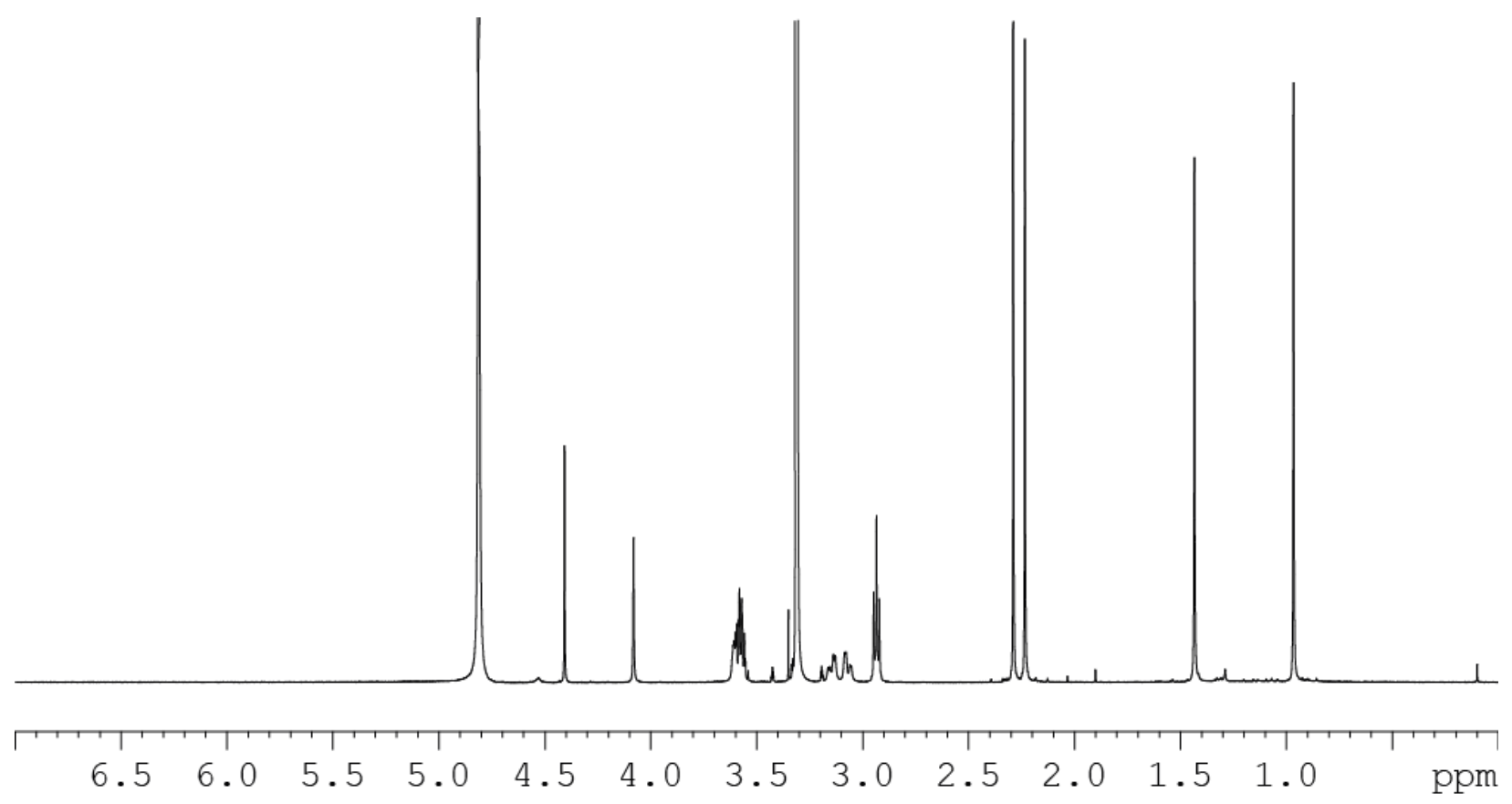

Figure S28: ${ }^{1} \mathrm{H}$ NMR (methanol- $d_{4}, 600 \mathrm{MHz}$ ) spectrum of $\mathbf{1 a}$. 


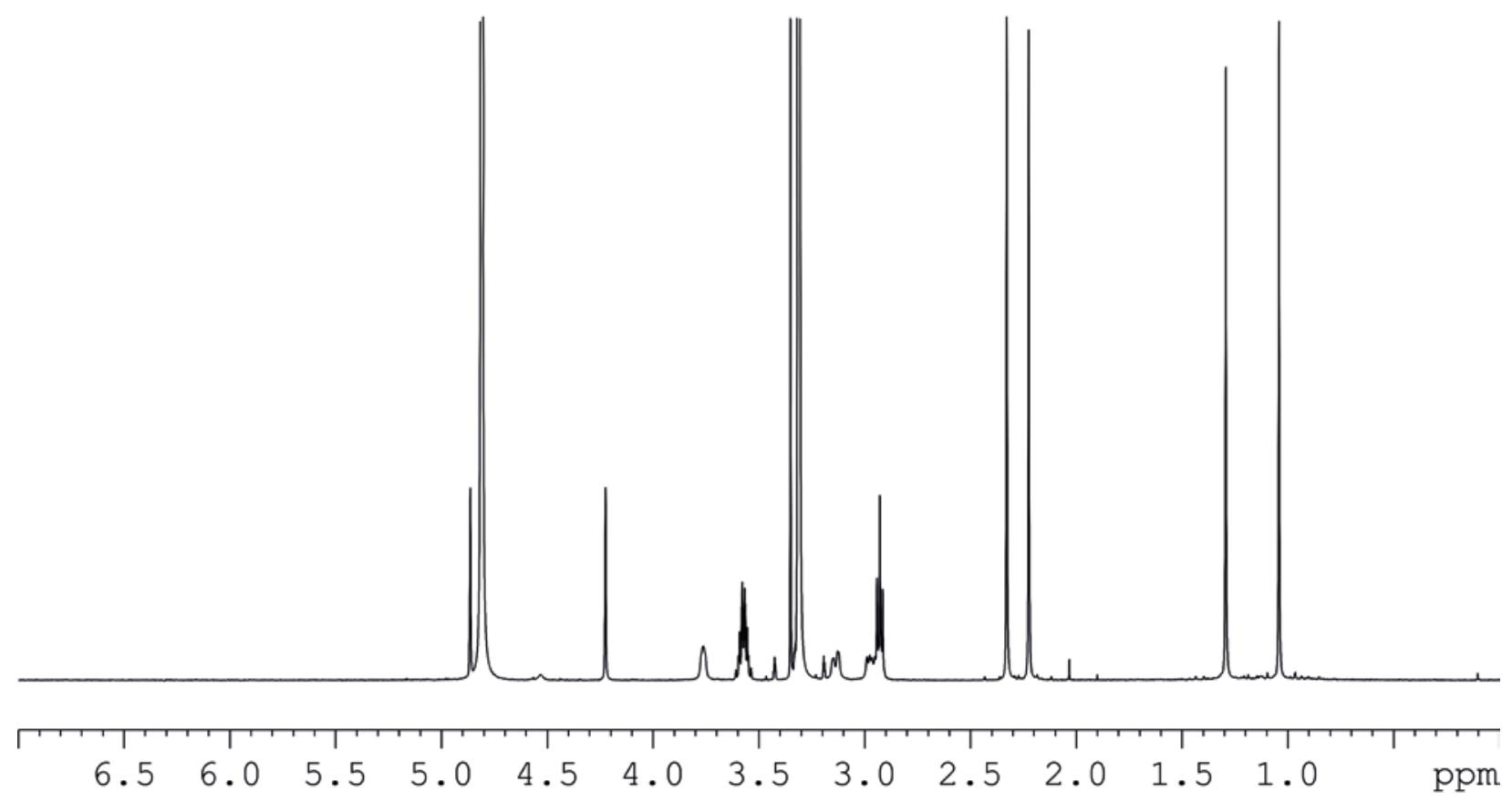

Figure S29: ${ }^{1} \mathrm{H}$ NMR (methanol- $d_{4}, 600 \mathrm{MHz}$ ) spectrum of $\mathbf{1 b}$.

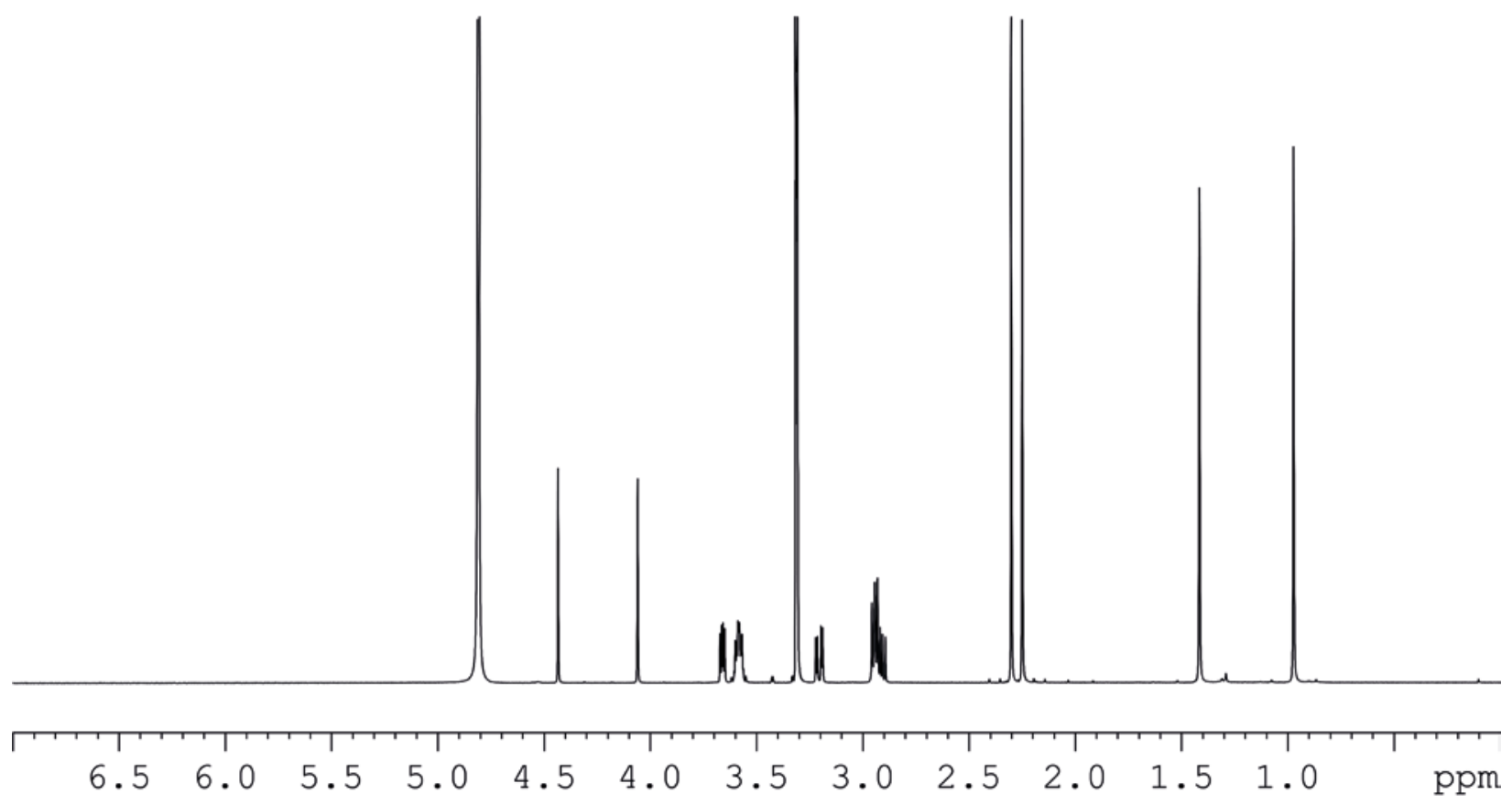

Figure S30: ${ }^{1} \mathrm{H}$ NMR (methanol- $d_{4}, 600 \mathrm{MHz}$ ) spectrum of $\mathbf{2 b}$. 

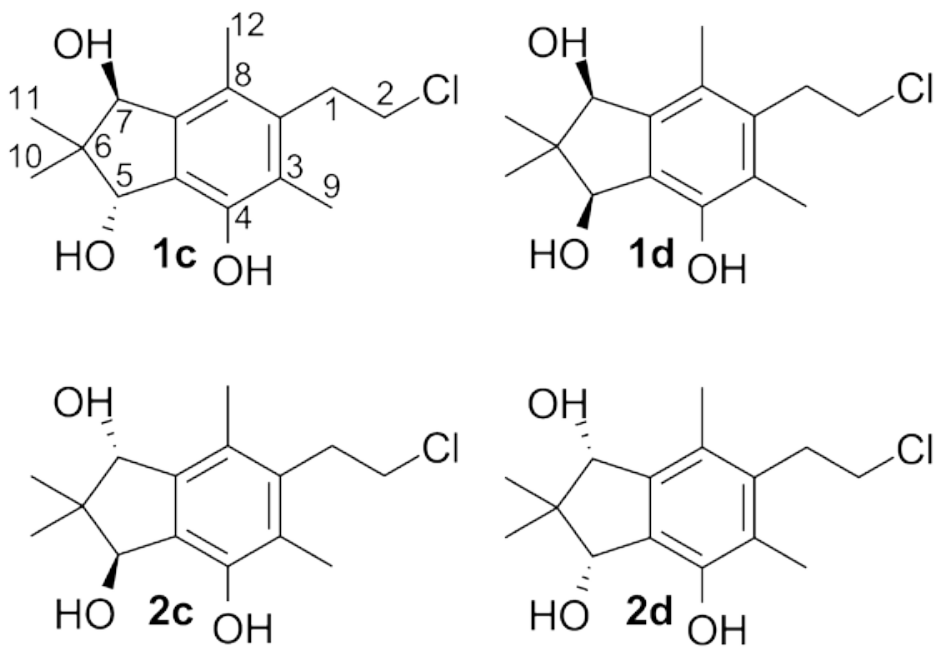

Figure S31: Structure of compound 1c, 1d, 2c and 2d.

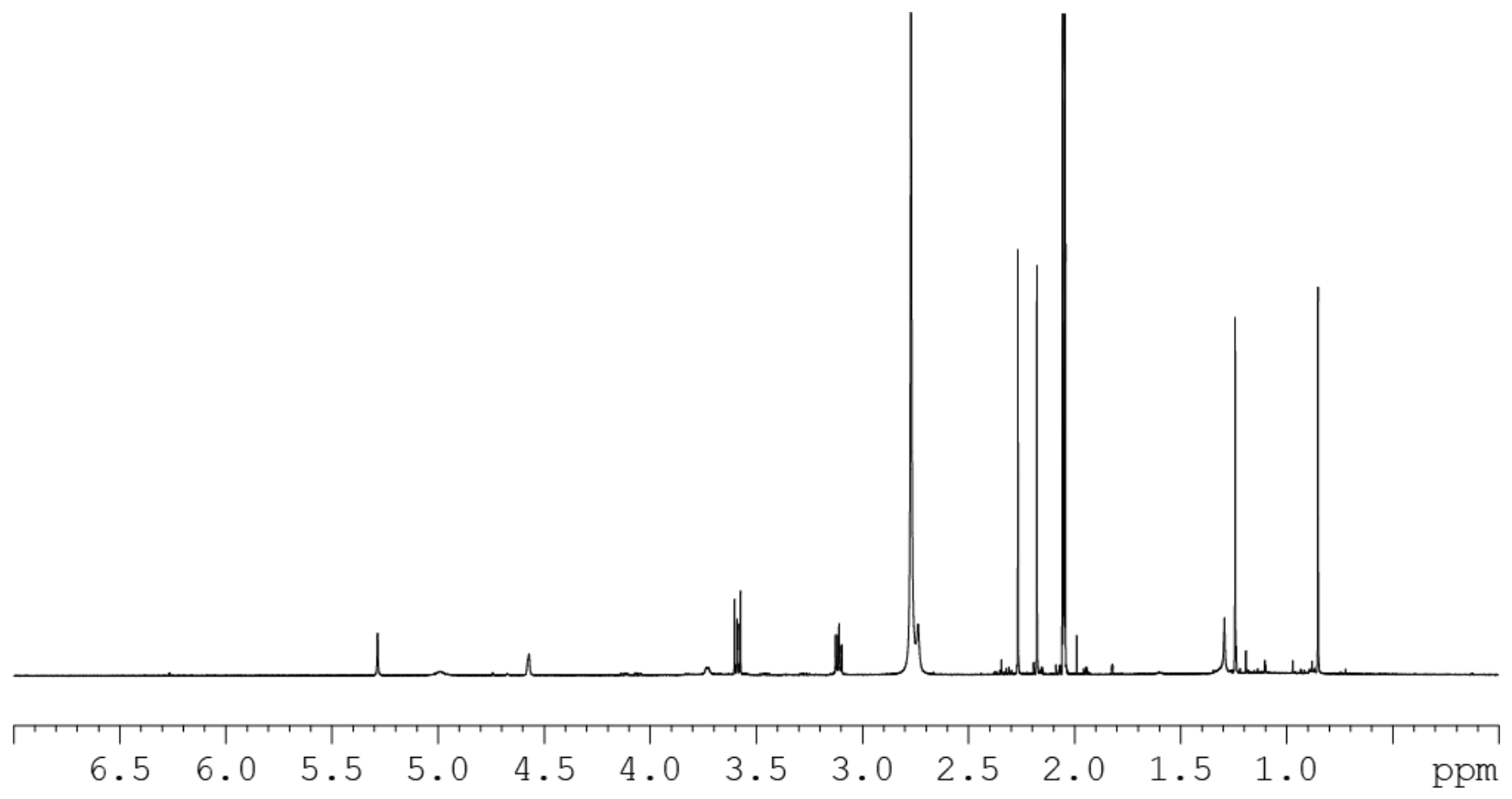

Figure S32: ${ }^{1} \mathrm{H}$ NMR (acetone- $d_{6}, 600 \mathrm{MHz}$ ) spectrum of $1 \mathrm{c}$. 


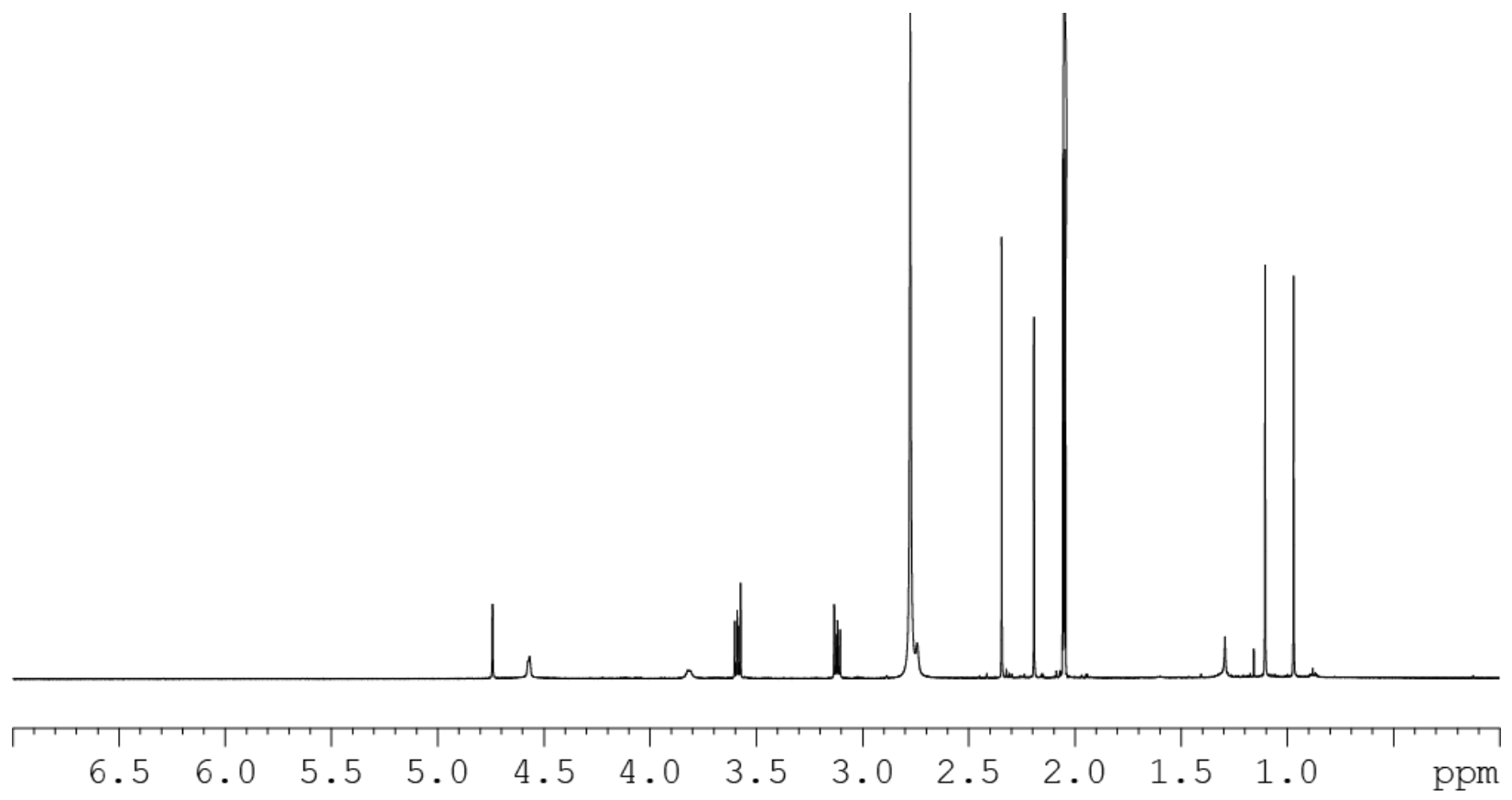

Figure S33: ${ }^{1} \mathrm{H}$ NMR (acetone- $d_{6}, 600 \mathrm{MHz}$ ) spectrum of 1 d.

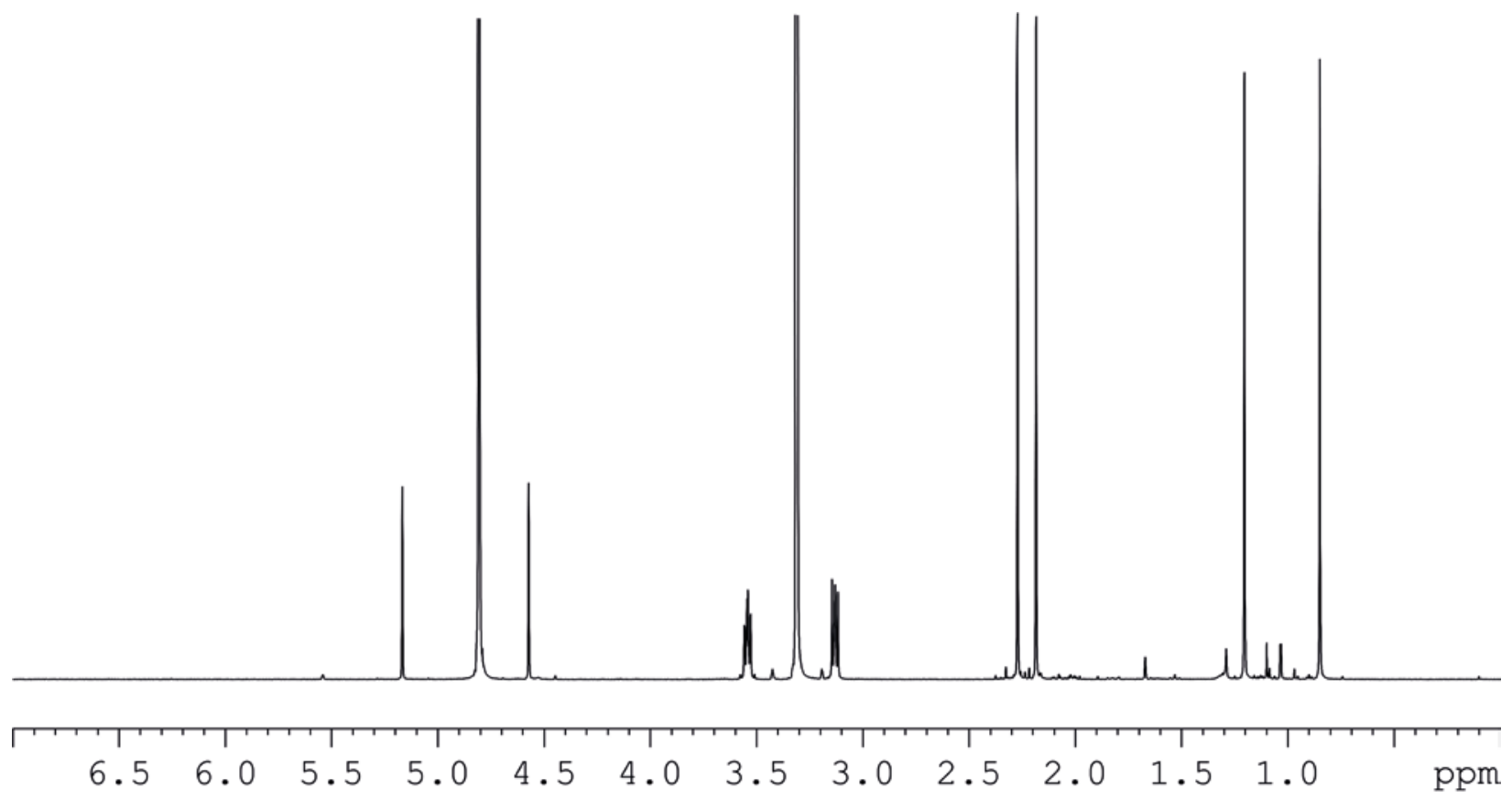

Figure S34: $1 \mathrm{H}$ NMR (methanol- $d_{4}, 600 \mathrm{MHz}$ ) spectrum of 2c. 


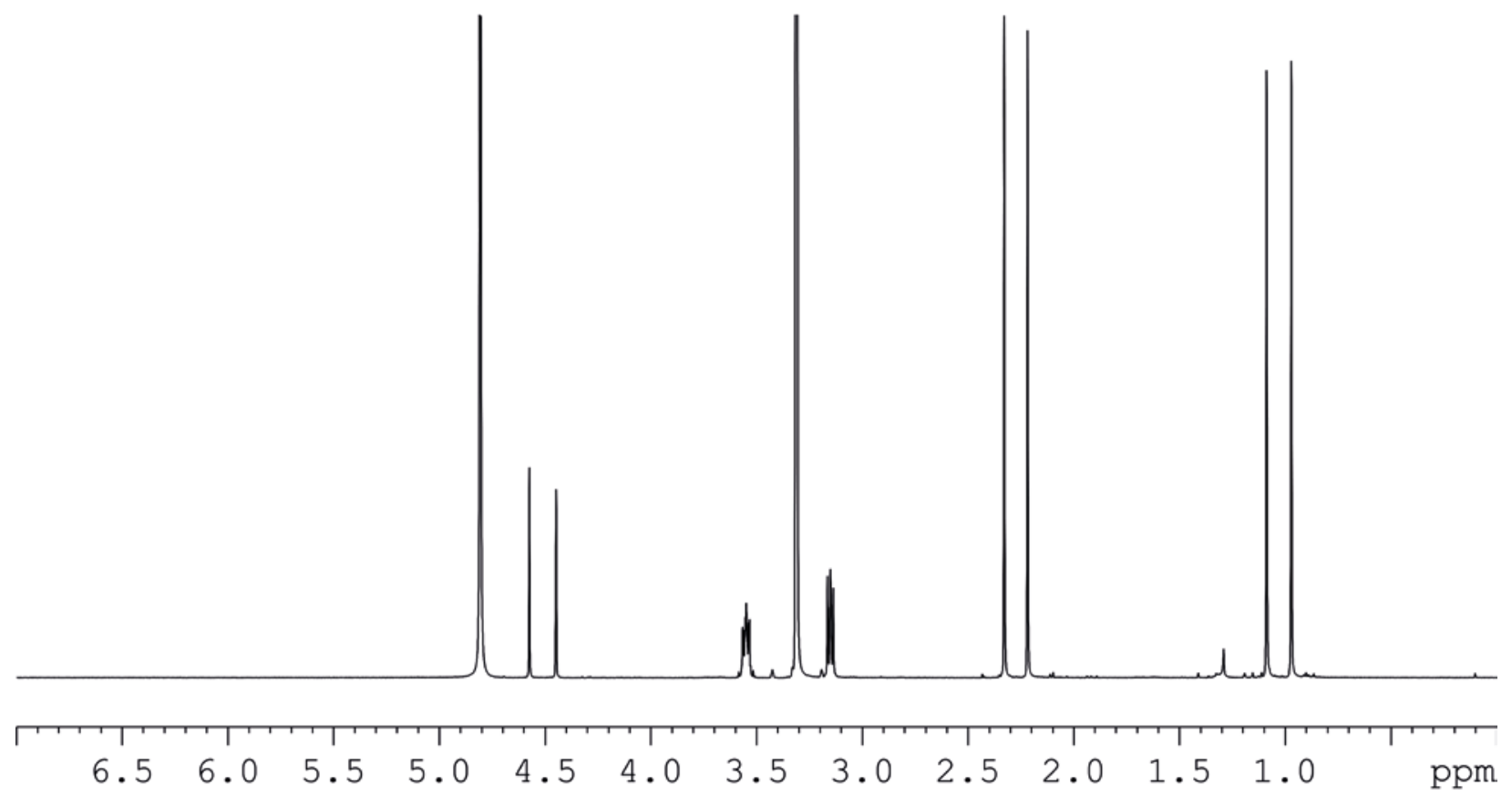

Figure S35: $1 \mathrm{H}$ NMR (methanol- $d_{4}, 600 \mathrm{MHz}$ ) spectrum of 2d. 
Table 1S. ${ }^{1} \mathrm{H}-\mathrm{NMR}$ data for compounds $2 \mathbf{2}$ and 3a in methanol- $d_{4}, \mathbf{5}$ in DMSO- $d_{6}$ and $\mathbf{6}$ in aceton- $d_{6}(600 \mathrm{MHz})$.

\begin{tabular}{|c|c|c|c|c|}
\hline & $\underline{2 a}$ & $\underline{3 a}$ & $\underline{5}$ & $\underline{6}$ \\
\hline pos. & $\delta_{\underline{\underline{H}}}(J$ in $\mathrm{Hz})$ & $\delta_{\underline{\underline{H}}}(J$ in $\mathrm{Hz})$ & $\underline{\delta}_{\underline{\mathrm{H}}}(\mathrm{J}$ in $\mathrm{Hz})$ & $\delta_{\underline{H}}(J$ in $\mathrm{Hz})$ \\
\hline 1 & $0.87, \mathrm{~m}$ & 1.09, ddd $(9.9,6.0,4.0)$ & $0.78, \mathrm{~m}$ & 1.07, ddd $(9.9,6.0,3.6)$ \\
\hline & $0.54, \mathrm{~m}$ & 0.47 , ddd $(9.6,6.8,4.0)$ & $0.69, \mathrm{~m}$ & 0.43 , ddd $(9.6,6.5,3.6)$ \\
\hline 2 & $0.80, \mathrm{~m}$ & 1.01 , ddd $(9.6,6.0,4.5)$ & $0.71, \mathrm{~m}$ & $0.86, \mathrm{~m}$ \\
\hline & $0.55, \mathrm{~m}$ & 0.79 , ddd $(9.9,6.8,4.5)$ & $0.67, \mathrm{~m}$ & $0.84, \mathrm{~m}$ \\
\hline $\begin{array}{l}2 \mathrm{a} \\
3\end{array}$ & & & & \\
\hline 4 & & & $5.92, \mathrm{~s}$ & 5.99, m \\
\hline $\begin{array}{l}4 a \\
5 \\
6\end{array}$ & $4.16, \mathrm{~s}$ & $6.35, \mathrm{~s}$ & $5.23, \mathrm{~s}$ & $5.15, \mathrm{~d}(2.6)$ \\
\hline 7 & 4.26, s & $4.68, \mathrm{~s}$ & $\begin{array}{l}2.01, \mathrm{~d}(12.6) \\
1.44, \mathrm{~d}(12.6)\end{array}$ & $\begin{array}{l}\text { 1.98, dd }(13.0,7.8) \\
1.43 \text {, dd }(13.0,9.3)\end{array}$ \\
\hline $7 a$ & & & $3.79, \mathrm{~s}, \mathrm{OH}$ & $3.00, \mathrm{~m}$ \\
\hline 8 & & & $3.69, \mathrm{~s}, \mathrm{OH}$ & $3.12, \mathrm{~m}, \mathrm{OH}$ \\
\hline 9 & $1.34, \mathrm{~s}$ & $1.30, \mathrm{~s}$ & $1.44, \mathrm{~s}$ & $1.52, \mathrm{~d}(1.4)$ \\
\hline 10 & $1.07, \mathrm{~s}$ & $1.15, \mathrm{~s}$ & $1.18, \mathrm{~s}$ & $1.10, \mathrm{~s}$ \\
\hline 11 & $0.95, \mathrm{~s}$ & 3.99, $\mathrm{m}$ & 1.03, s & 3.33, $\mathrm{m}$ \\
\hline 12 & $1.45, \mathrm{~s}$ & $1.72, \mathrm{~s}$ & $0.80, \mathrm{~s}$ & $3.40, \mathrm{~m}, \mathrm{OH}$ \\
\hline 13 & & & & \\
\hline 14 & & $2.01, \mathrm{~s}$ & & \\
\hline
\end{tabular}


Table 2S. ${ }^{1} \mathrm{H}-\mathrm{NMR}$ data for the S- and R-MTPA monoesters of $\mathbf{1}, 2$ and 3a (Pyridine- $d_{5}, 30^{\circ} \mathrm{C}$ ).

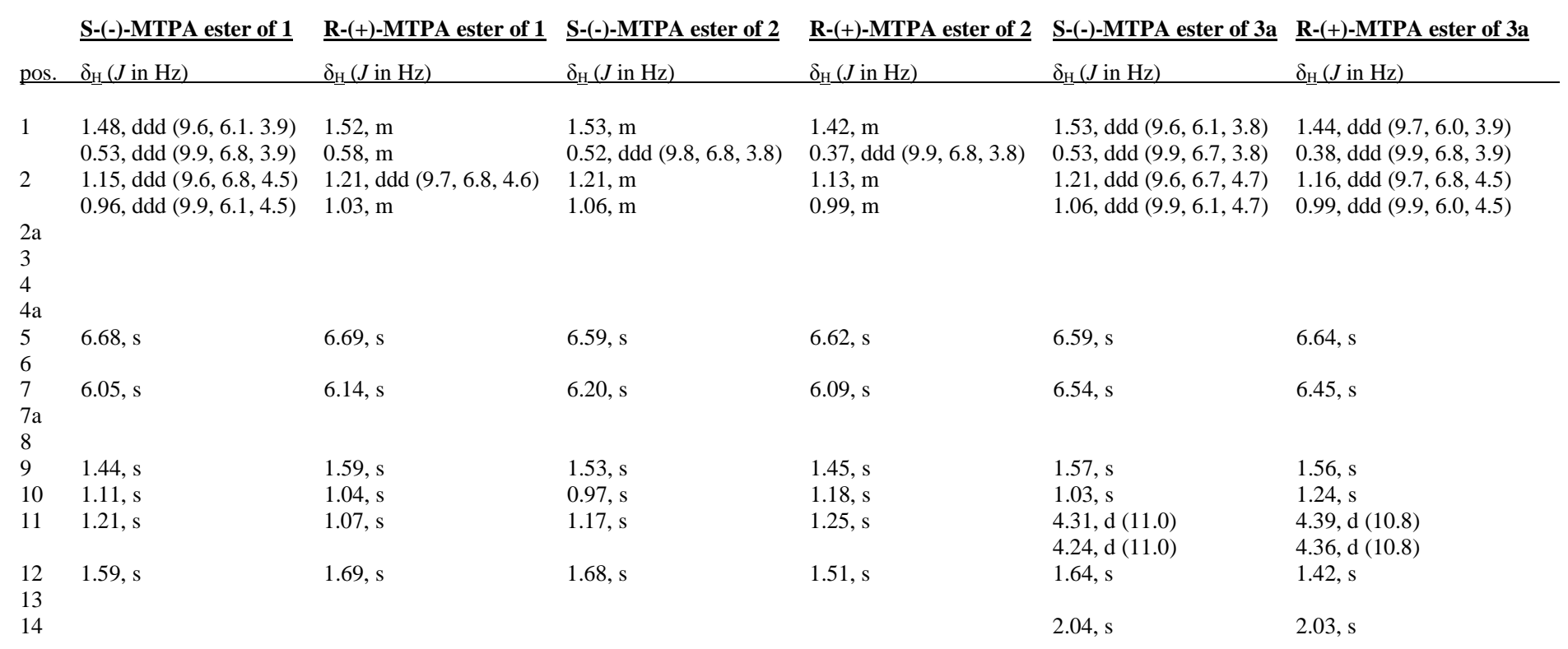


Table 3S. ${ }^{1} \mathrm{H}-\mathrm{NMR}$ data for compound $\mathbf{1 a}, \mathbf{1 b}, \mathbf{2 b}, \mathbf{2 c}$ and $2 \mathbf{d}$ in methanol- $d_{4}$ and for $\mathbf{1 c}$ and $\mathbf{1 d}$ in aceton- $d_{6}(600 \mathrm{MHz})$.

\begin{tabular}{|c|c|c|c|c|c|c|c|}
\hline & $\underline{1 a}$ & $1 b$ & $1 \mathrm{c}$ & 1d & $2 \mathbf{b}$ & $2 \mathrm{c}$ & $2 \mathbf{d}$ \\
\hline pos. & $\delta_{\underline{H}}(J$ in Hz) & $\delta_{\underline{H}}(J$ in Hz $)$ & $\delta_{\underline{H}}(J$ in Hz $)$ & $\underline{\delta}_{\underline{H}}(J$ in Hz) & $\underline{\delta}_{\underline{H}}(J$ in Hz) & $\delta_{\underline{H}}(J$ in Hz $)$ & $\delta_{\underline{H}}(J$ in $\mathrm{Hz})$ \\
\hline 1 & 2.93, m & 2.93, m & $3.11, \mathrm{~m}$ & 3.12, m & $2.94, \mathrm{~m}$ & 3.13, m & $3.15, \mathrm{~m}$ \\
\hline $\begin{array}{l}2 \\
2 \mathrm{a}\end{array}$ & $3.58, \mathrm{~m}$ & $3.57, \mathrm{~m}$ & 3.59, m & $3.59, \mathrm{~m}$ & 3.58, m & $3.54, \mathrm{~m}$ & $3.55, \mathrm{~m}$ \\
\hline $\begin{array}{l}3 \\
4\end{array}$ & & & & & & & \\
\hline $\begin{array}{l}5 \\
6\end{array}$ & 4.08, s & $4.22, \mathrm{~s}$ & $5.28, \mathrm{~s}$ & 4.74, s & 4.06, s & 5.17, s & 4.57, s \\
\hline $\begin{array}{l}7 \\
8\end{array}$ & 4.41, s & 4.86, s & 4.75 , br s & 4.57 , br s & 4.43, s & 4.57, s & 4.45, s \\
\hline 9 & $2.23, \mathrm{~s}$ & $2.22, \mathrm{~s}$ & 2.18, s & 2.19, s & $2.25, \mathrm{~s}$ & $2.19, \mathrm{~s}$ & 2.22, s \\
\hline 10 & 1.44, s & 1.29, s & $1.24, \mathrm{~s}$ & 1.10, s & 1.41, s & $1.21, \mathrm{~s}$ & 1.09, s \\
\hline 11 & $0.97, \mathrm{~s}$ & $1.04, \mathrm{~s}$ & $0.85, \mathrm{~s}$ & 0.97, s & 0.97, s & $0.85, \mathrm{~s}$ & 0.97, s \\
\hline 12 & $2.29, \mathrm{~s}$ & $2.33, \mathrm{~s}$ & 2.27, s & 2.34, s & $2.30, \mathrm{~s}$ & $2.27, \mathrm{~s}$ & 2.33, s \\
\hline
\end{tabular}

